MATHEMATICS OF COMPUTATION

Volume 80, Number 274, April 2011, Pages 1135-1154

S $0025-5718(2010) 02432-9$

Article electronically published on November 1, 2010

\title{
SUBIDEAL BORDER BASES
}

\author{
MARTIN KREUZER AND HENK POULISSE
}

\begin{abstract}
In modeling physical systems, it is sometimes useful to construct border bases of 0-dimensional polynomial ideals which are contained in the ideal generated by a given set of polynomials. We define and construct such subideal border bases, provide some basic properties and generalize a suitable variant of the Buchberger-Möller algorithm as well as the AVI-algorithm of Heldt, Kreuzer, Pokutta, and Poulisse to the subideal setting. The subideal version of the AVI-algorithm is then applied to an actual industrial problem.
\end{abstract}

\section{INTRODUCTION}

In [5] an algorithm was introduced which computes an approximate border basis consisting of unitary polynomials that vanish approximately at a given set of points. It has been shown that this AVI-algorithm is useful for modeling physical systems based on a set of measured data points. More precisely, given a finite point set $\mathbb{X}=\left\{p_{1}, \ldots, p_{s}\right\} \subset[-1,1]^{n}$, the AVI-algorithm computes an order ideal $\mathcal{O}$ of terms in $\mathbb{T}^{n}$ and an $\mathcal{O}$-border prebasis $G=\left\{g_{1}, \ldots, g_{\nu}\right\}$ such that

(1) the unitary polynomials $g_{i} /\left\|g_{i}\right\|$ vanish $\varepsilon$-approximately at $\mathbb{X}$, where $\varepsilon>0$ is a given threshold number, and

(2) the normal remainders of the S-polynomials $S\left(g_{i}, g_{j}\right)$ for $g_{i}, g_{j}$ with neighboring border terms are smaller than $\varepsilon$.

Abstractly speaking, the last condition means that the point in the moduli space corresponding to $G$ is "close" to the border basis scheme (see [11] and [8]). In practical applications, the AVI-algorithm turns out to be very stable and useful. With a judicious choice of the threshold number $\varepsilon$, it is able to discover simple polynomial relations which exist in the data with high reliability. For instance, it discovers simple physical laws inherent in measured data without the need of imposing model equations.

However, in some situations physical information may be available which is not contained in the data points $\mathbb{X}$ or we may have exact physical knowledge which is only approximately represented by the data points. An example for this phenomenon will be discussed in Section 6. For instance, we may want to impose certain vanishing conditions on the model equations we are constructing. Using Hilbert's Nullstellensatz this translates to saying that what we are looking for is the intersection of the vanishing ideal of $\mathbb{X}$ with a given ideal $J \subseteq \mathbb{R}\left[x_{1}, \ldots, x_{n}\right]$ whose generators represent the vanishing conditions we want to impose.

Received by the editor May 7, 2009 and, in revised form, March 9, 2010.

2010 Mathematics Subject Classification. Primary 13P10; Secondary 41A10, 65D05, 14Q99.

Key words and phrases. Approximate vanishing ideal, Buchberger-Möller algorithm, border basis.

(c) 2010 American Mathematical Society Reverts to public domain 28 years from publication 
In order to be able to deal with this approximate situation, it is first necessary to generalize the exact version of the computation of vanishing ideals to the subideal setting. Then this theory will serve as a guide and a motivation for the approximate case. Therefore this paper begins in Section 2 with the definition and basic properties of subideal border bases.

Given a 0 -dimensional ideal $I$ in a polynomial ring $P=K\left[x_{1}, \ldots, x_{n}\right]$ over a field and a set of polynomials $F=\left\{f_{1}, \ldots, f_{m}\right\}$ generating an ideal $J=\langle F\rangle$, a subideal border basis of $I$ corresponds to a set of polynomials $\mathcal{O}_{F}=\mathcal{O}_{1} \cdot f_{1} \cup \cdots \cup \mathcal{O}_{m} \cdot f_{m}$, where the $\mathcal{O}_{i}$ are order ideals of terms, such that the residue classes of the elements of $\mathcal{O}_{F}$ form a $K$-basis of $J /(I \cap J) \cong(I+J) / I$. Clearly, this generalizes the case $F=\{1\}$, i.e. the "usual" border basis theory. We examine the question whether subideal border bases always exist and explain a method to construct them from a border basis of $I$. Moreover, we discuss some uniqueness properties of subideal border bases.

The foundation of any further development of the theory of subideal border bases is a generalization of the Border Division Algorithm (see [10, 6.4.11) to the subideal case. This foundation is laid in Section 3 where we also study higher $\mathcal{O}_{F}$-borders, the $\mathcal{O}_{F}$-index, and show that a subideal border basis of $I$ generates $I \cap J$.

In Section 4, we generalize the Buchberger-Möller algorithm (BM-algorithm) for computing vanishing ideals of point sets to the subideal setting. More precisely, we generalize a version of the BM-algorithm which proceeds blockwise degree-bydegree and produces a border basis of the vanishing ideal. Similarly, the subideal version of the BM-algorithm (cf. Algorithm 4.2) computes an $\mathcal{O}_{\sigma}\left(I_{\mathbb{X}}\right)_{F}$-subideal border basis of $I_{\mathbb{X}}$, where $\mathcal{O}_{\sigma}\left(I_{\mathbb{X}}\right)$ is the complement of a leading term ideal of the vanishing ideal $I_{\mathbb{X}}$ of $\mathbb{X}$.

Next, in Section 5, we turn to the setting of Approximate Computational Algebra. We work in the polynomial ring $\mathbb{R}\left[x_{1}, \ldots, x_{n}\right]$ over the reals and assume that $\mathbb{X} \subset[-1,1]^{n}$ is a finite set of (measured, imprecise) points. We define approximate $\mathcal{O}_{F}$-subideal border bases and generalize the AVI-algorithm from [5], Thm. 3.3 to the subideal case. A discussion of the efficiency and some timings for the implementations of the subideal versions of the BM-algorithm and the AVI-algorithm in ApCoCoA (cf. [3]) are provided in Section 6.

Let us point out that the subideal version of the AVI-algorithm contains a substantial difference to the traditional way of computing approximate vanishing ideals, e.g., as in [1]. Namely, the AVI-algorithm produces a set of polynomials which vanish approximately at the given data points, but we do not demand that there exists a "nearby" set of points at which these polynomials vanish exactly. The latter requirement has turned out to be too restrictive for real-world applications, for instance, the one we explain in the last section. There we provide an example for the application of these techniques to the problem of production allocation in the oil industry.

Unless explicitly stated otherwise, we use the notation and definitions of 9] and [10. We shall assume that the reader has some familiarity with the theory of exact and approximate border bases (see for instance [5], 6], 7], 8], Section 6.4 of [10], and [13]). 


\section{SUBIDEAL BORDER BASES}

Here we are interested in a "relative" version of the notion of border bases in the following sense. Let $K$ be a field, let $P=K\left[x_{1}, \ldots, x_{n}\right]$ be a polynomial ring, let $\mathbb{T}^{n}$ be its monoid of terms, let $\mathcal{O}$ be an order ideal in $\mathbb{T}^{n}$, and let $I \subset P$ be a 0-dimensional ideal.

Suppose we are given a further polynomial ideal $J=\left\langle f_{1}, \ldots, f_{m}\right\rangle$ of $P$, where $F=\left\{f_{1}, \ldots, f_{m}\right\} \subset P \backslash\{0\}$. Our goal is to describe and compute the intersection ideal $I \cap J$ as a subideal of $J$. By Noether's isomorphism theorem, we have $J /(I \cap$ $J) \cong(I+J) / I \subset P / I$. Therefore, $J$ has a finite $K$-vector space basis modulo $I \cap J$. Now we are looking for the following special kind of vector space basis.

Definition 2.1. Let $I, J$ be ideals of $P$ as above, and let $\mathcal{O}$ be an order ideal of terms in $\mathbb{T}^{n}$ whose residue classes form a $K$-vector space basis of $P / I$.

(1) For $i=1, \ldots, m$, let $\mathcal{O}_{i} \subseteq \mathcal{O}$ be an order ideal. Then the set $\mathcal{O}_{F}=$ $\mathcal{O}_{1} \cdot f_{1} \cup \cdots \cup \mathcal{O}_{m} \cdot f_{m}$ is called an $F$-order ideal. Its elements, i.e., products of the form $t f_{i}$ with $t \in \mathcal{O}_{i}$ will be called $F$-terms.

(2) If $\mathcal{O}_{F}=\mathcal{O}_{1} \cdot f_{1} \cup \cdots \cup \mathcal{O}_{m} \cdot f_{m}$ is an $F$-order ideal whose residue classes form a $K$-vector space basis of $J /(I \cap J)$, we say that the ideal $I$ has an $\mathcal{O}_{F}$-subideal border basis.

Notice that an $F$-term may be viewed as a generalization of the usual notion of term by using $F=\{1\}$. Similarly, $F$-order ideals generalize the usual order ideals. It is natural to ask whether every ideal $I$ supporting an $\mathcal{O}$-border bases has an $\mathcal{O}_{F}$-subideal border basis for some $F$-order ideal $\mathcal{O}_{F}$. The next example answers this question negatively.

Example 2.2. Let $P=\mathbb{Q}[x, y]$, and let

$$
I=\left\langle(x-y)(y-1),(x-y)(x+y-1), y^{3}-y\right\rangle
$$

be the vanishing ideal of $\mathbb{X}=\{(0,0),(1,1),(-1,-1),(0,1)\}$. The ideal $I$ has a border basis with respect to the order ideal $\mathcal{O}=\{1, x, y, x y\}$.

Now we consider the set $F=\{f\}$ where $f=x-y+1$. The ideal $\bar{J}=\langle\bar{f}\rangle$ in $P / I$ is a 3 -dimensional $\mathbb{Q}$-vector space. The only 3 -element order ideal contained in $\mathcal{O}$ is $\mathcal{O}^{\prime}=\{1, x, y\}$. However, we have $(x-y) \bar{f}=0$ in $P / I$. Therefore $\mathcal{O}^{\prime}$ is not a $\mathbb{Q}$-vector space basis of $J /(I \cap J)$, and the ideal $I$ has no $\mathcal{O}_{F}$-subideal border basis.

Let us explain this result geometrically. The annihilator of the ideal $J$ is the 1-dimensional ideal $\langle\bar{g}\rangle$ of $P / I$ generated by the separator $g=y-x$ of the point $(0,1)$ in $\mathbb{X}$. In order to get an $\mathcal{O}_{F}$-subideal border basis of $I$ we should remove $x$ or $y$ from $\mathcal{O}$, but this destroys the order ideal property. The fact that $g$ does not contain $x y$ in its support is due to the failure of the Cayley-Bacharach property for $\mathbb{X}$.

In practice, almost all ideals $I$ have $\mathcal{O}_{F}$-subideal border bases for all possible sets $F$. The following definition provides the essential property.

Definition 2.3. Let $I \subset P$ be a 0 -dimensional ideal which has an $\mathcal{O}$-border basis. We say that $I$ is $\mathcal{O}$-uniform if every ideal $I^{\prime} \subset P$ containing $I$ has an $\mathcal{O}^{\prime}$-border basis with respect to an order ideal $\mathcal{O}^{\prime}$ contained in $\mathcal{O}$.

Our next remark yields a large class of $\mathcal{O}$-uniform ideals. 
Remark 2.4. Let $\sigma$ be a term ordering, and let $\mathcal{O}=\mathbb{T}^{n} \backslash \operatorname{LT}_{\sigma}(I)$. Then the ideal $I$ has an $\mathcal{O}$-border basis by [10, 6.4.18. For every ideal $I^{\prime} \subset P$ containing $I$, the order ideal $\mathcal{O}^{\prime}=\mathbb{T}^{n} \backslash \operatorname{LT}_{\sigma}\left(I^{\prime}\right)$ is contained in $\mathcal{O}$, and $I^{\prime}$ has an $\mathcal{O}^{\prime}$-border basis. Thus the ideal $I$ is $\mathcal{O}$-uniform.

Now we show that $\mathcal{O}$-uniform ideals have $\mathcal{O}_{F}$-subideal border bases for all sets $F$.

Proposition 2.5. Let $I \subset P$ be a 0-dimensional ideal, let $f_{1}, \ldots, f_{m} \in P \backslash\{0\}$, and let $J=\left\langle f_{1}, \ldots, f_{m}\right\rangle$. If $I$ has an $\mathcal{O}$-border basis and $I$ is $\mathcal{O}$-uniform, then there exist order ideals $\mathcal{O}_{i} \subseteq \mathcal{O}$ such that the residue classes of $\mathcal{O}_{F}=\mathcal{O}_{1} f_{1} \cup \cdots \cup \mathcal{O}_{m} f_{m}$ are a $K$-vector space basis of $J /(I \cap J)$. In other words, the ideal $I$ has an $\mathcal{O}_{F}$-subideal border basis.

Proof. To show the claim, we proceed by induction on $m$. The ideal $\left\langle\bar{f}_{1}\right\rangle$ in $P / I$ is isomorphic to $(P / I) / \operatorname{Ann}\left(\bar{f}_{1}\right)$. Since $I$ is $\mathcal{O}$-uniform, there is an order ideal $\mathcal{O}_{1}$ contained in $\mathcal{O}$ whose elements are a $K$-basis of $(P / I) / \operatorname{Ann}\left(\bar{f}_{1}\right)$. Under the isomorphism, this $K$-basis is mapped to $\mathcal{O}_{1} \cdot \bar{f}_{1}$.

Now we prove the induction step. By the inductive hypothesis, there exist order ideals $\mathcal{O}_{i} \subseteq \mathcal{O}$ for $i=1, \ldots, m-1$ such that $\mathcal{O}_{1} \cdot f_{1} \cup \cdots \cup \mathcal{O}_{m-1} \cdot f_{m-1}$ represents a $K$-basis of the ideal $\left\langle\bar{f}_{1}, \ldots, \bar{f}_{m-1}\right\rangle$ in $P / I$. This ideal is a $K$-vector subspace of $(I+J) / I=\left\langle\bar{f}_{1}, \ldots, \bar{f}_{m}\right\rangle$. Therefore it suffices to find an order ideal $\mathcal{O}_{m}$ contained in $\mathcal{O}$ such that the residue classes of its elements are a $K$-basis of the ideal $\left\langle\bar{f}_{m}\right\rangle$ in $P^{\prime}=P /\left(I+\left\langle\bar{f}_{1}, \ldots, \bar{f}_{m-1}\right\rangle\right)$. Again we use the fact that $I$ is $\mathcal{O}$-uniform to get an order ideal $\mathcal{O}^{\prime}$ contained in $\mathcal{O}$ whose elements are a $K$-basis of $P^{\prime}$. Considering the isomorphism $\left\langle\bar{f}_{m}\right\rangle \cong P^{\prime} / \operatorname{Ann}\left(\bar{f}_{m}\right)$, the claim follows as for $m=1$.

In the setting of this proposition, we can construct an $F$-order ideal such that a given ideal $I$ has an $\mathcal{O}_{F}$-subideal border basis. The following example illustrates the method.

Example 2.6. Let $P=\mathbb{Q}[x, y]$, let $I=\left\langle x^{2}-x, y^{2}-y\right\rangle$, let $\mathcal{O}=\{1, x, y, x y\}$, and let $J=\langle x+y\rangle$. Then $I$ has an $\mathcal{O}$-border basis and therefore also an $\mathcal{O}_{F}$-subideal border basis w.r.t. $F=\{f\}$ for $f=x+y$.

To construct a suitable $F$-order ideal, we start with $\mathcal{O}_{F}=\{1 \cdot f\}$. Then we put $x \cdot f$ and $y \cdot f$ into $\mathcal{O}_{F}$, since we have $x \cdot f \equiv x y+x$ and $y \cdot f \equiv x y+y$ modulo $I$, and since $\{x+y, x y+x, x y+y\}$ is $\mathbb{Q}$-linearly independent in $P / I$. Next, $x y \cdot f \equiv 2 x y \equiv x \cdot f+y \cdot f-1 \cdot f$ implies that we are done. The result is that $\mathcal{O}_{F}=\{f, x f, y f\}$ is an $F$-order ideal for which $I$ has an $\mathcal{O}_{F}$-subideal border basis.

At this point it is time to explain the choice of the term "subideal border basis" in the above definition.

Definition 2.7. Let $F=\left\{f_{1}, \ldots, f_{m}\right\} \subset P \backslash\{0\}$, let $J=\left\langle f_{1}, \ldots, f_{m}\right\rangle$ be the ideal generated by $F$, and let $\mathcal{O}_{F}=\mathcal{O}_{1} f_{1} \cup \cdots \cup \mathcal{O}_{m} f_{m}$ be an $F$-order ideal. We write $\mathcal{O}_{F}=\left\{t_{1} f_{\alpha_{1}}, \ldots, t_{\mu} f_{\alpha_{\mu}}\right\}$ with $\alpha_{i} \in\{1, \ldots, m\}$ and $t_{i} \in \mathcal{O}_{\alpha_{i}}$.

(1) The set of all polynomials $x_{i} t_{j} f_{\alpha_{j}}$ such that $i \in\{1, \ldots, n\}, j \in\{1, \ldots, \mu\}$ and $\left(x_{i} t_{j}\right) f_{\alpha_{j}} \notin \mathcal{O}_{\alpha_{j}} f_{\alpha_{j}}$ is called the border of $\mathcal{O}_{F}$ and denoted by $\partial \mathcal{O}_{F}$.

(2) Let $\partial \mathcal{O}_{F}=\left\{b_{1} f_{\beta_{1}}, \ldots, b_{\nu} f_{\beta_{\nu}}\right\}$. A tuple of polynomials $G=\left(g_{1}, \ldots, g_{\nu}\right)$ is called an $\mathcal{O}_{F}$-subideal border prebasis if $g_{j}=b_{j} f_{\beta_{j}}-\sum_{i=1}^{\mu} c_{i j} t_{i} f_{\alpha_{i}}$ with $c_{1 j}, \ldots, c_{\mu j} \in K$ for $j=1, \ldots, \nu$.

(3) An $\mathcal{O}_{F}$-subideal border prebasis $G$ is called an $\mathcal{O}_{F}$-subideal border basis of an ideal $I$ if the elements of $G$ are contained in $I$ and the residue classes of the elements of $\mathcal{O}_{F}$ form a $K$-vector space basis of $J /(I \cap J)$. 
In this terminology, the preceding proposition can be rephrased as follows.

Corollary 2.8. Let $\mathcal{O}$ be an order ideal in $\mathbb{T}^{n}$, and let $I \subset P$ be a 0-dimensional ideal which has an $\mathcal{O}$-border basis and which is $\mathcal{O}$-uniform. Then $I$ has an $\mathcal{O}_{F-}$ subideal border basis for every ideal $J=\left\langle f_{1}, \ldots, f_{m}\right\rangle$ and $F=\left\{f_{1}, \ldots, f_{m}\right\} \subset$ $P \backslash\{0\}$.

In the setting of Example 2.6 the $\mathcal{O}_{F}$-subideal border basis of $I$ can be constructed as follows.

Example 2.9. The border of the $F$-order ideal $\mathcal{O}_{F}=\{f, x f, y f\}$ is $\partial \mathcal{O}_{F}=$ $\left\{x^{2} f, x y f, y^{2} f\right\}$. We compute modulo $I$ and find $x^{2} f \equiv x f, x y f \equiv x f+y f-f$, and $y^{2} f \equiv y f$. Therefore the tuple $G=\left(x^{2} f-x f, x y f-x f-y f+f, y^{2} f-y f\right)$ is an $\mathcal{O}_{F}$-subideal border basis of $I$.

If an ideal has an $\mathcal{O}_{F}$-subideal border basis, the elements of this basis are uniquely determined. This follows exactly as in the case $J=\langle 1\rangle$, i.e., the case of the usual border bases (see [10, 6.4.17 and 6.4.18). Notice, however, that a set of polynomials may be an $F$-order ideal in several different ways. This is illustrated by the following example.

Example 2.10. Let $P=\mathbb{Q}[x, y]$, let $I=\left\langle x^{2}-x, y^{2}-y\right\rangle$, and let $J=\langle x, y\rangle$. Clearly, the ideal $I$ has an $\mathcal{O}$-border basis for $\mathcal{O}=\{1, x, y, x y\}$, namely the tuple $G=\left(x^{2}-x, x^{2} y-x y, x y^{2}-x y, y^{2}-y\right)$. Hence the ideal $I$ also has an $\mathcal{O}_{F}$-subideal border basis for $F=\{x, y\}$. Here we can use both $\mathcal{O}_{F}=\{1, y\} \cdot x \cup\{1\} \cdot y$ and $\mathcal{O}_{F}=\{1\} \cdot x \cup\{1, x\} \cdot y$.

This example shows also another phenomenon: a polynomial can simultaneously be contained in $\mathcal{O}_{F}$ and in $\partial \mathcal{O}_{F}$. For instance, if we use $\mathcal{O}_{F}=\{1, y\} \cdot x \cup\{1\} \cdot y$, the term $x y$ is both contained in $\{1, y\} \cdot x$ and in the border of $\{1\} \cdot y$. The resulting subideal border basis will contain the polynomial $x y-x y=0$.

Finally, we give an example where a term is in $\partial \mathcal{O}_{F}$ in two different ways, so that a subideal border basis polynomial is repeated.

Example 2.11. Let $I=\left\langle x^{2}-x, y^{2}-y, x y\right\rangle \subseteq \mathbb{Q}[x, y]$, and let $J=\langle x, y\rangle \subset \mathbb{Q}[x, y]$. Then the subideal border basis of $I$ with respect to $\mathcal{O}_{F}=\{1\} \cdot x \cup\{1\} \cdot y$ is $G=\left(x^{2}-x, x y, x y, y^{2}-y\right)$ where $x y$ appears both in $\partial\{1\} \cdot x$ and in $\partial\{1\} \cdot y$.

\section{The SubideAL BORDER DIVISION ALGORITHM}

A central result in the construction of any Gröbner basis-like theory is a suitable version of the division algorithm (for the classical case, see for instance 9 , Thm. 1.6.4 and specifically for border bases, see [10], Prop. 6.4.11). Before we can present a subideal border basis version, we need a few additional definitions.

Definition 3.1. Let $F=\left\{f_{1}, \ldots, f_{m}\right\} \subset P \backslash\{0\}$, and let $\mathcal{O}_{F}$ be an $F$-order ideal.

(1) First, we let $\partial^{0} \mathcal{O}_{F}=\overline{\partial^{0} \mathcal{O}_{F}}=\mathcal{O}_{F}$.

(2) For every $k \geq 1$, we inductively define the $k^{\text {th }}$ border of $\mathcal{O}_{F}$ by $\partial^{k} \mathcal{O}_{F}=$ $\partial\left(\overline{\partial^{k-1} \mathcal{O}_{F}}\right)$ and the $k^{\text {th }}$ border closure of $\mathcal{O}_{F}$ by $\overline{\partial^{k} \mathcal{O}_{F}}=\overline{\partial^{k-1} \mathcal{O}_{F}} \cup \partial^{k} \mathcal{O}_{F}$.

(3) In particular, the first border closure of $\mathcal{O}_{F}$ is $\overline{\partial \mathcal{O}_{F}}=\overline{\partial^{1} \mathcal{O}_{F}}=\mathcal{O}_{F} \cup \partial \mathcal{O}_{F}$.

Using these higher borders, the set $\mathbb{T}^{n} f_{1} \cup \cdots \cup \mathbb{T}^{n} f_{m}$ is partitioned as follows. 
Proposition 3.2. Let $F=\left\{f_{1}, \ldots, f_{m}\right\} \subset P \backslash\{0\}$, and let $\mathcal{O}_{F}$ be an $F$-order ideal.

(1) For every $k \geq 0$, we have a disjoint union $\overline{\partial^{k} \mathcal{O}_{F}}=\bigcup_{i=0}^{k} \partial^{i} \mathcal{O}_{F}$.

(2) For every $k \geq 0$, we have $\overline{\partial^{k} \mathcal{O}_{F}}=\mathbb{T}_{\leq k}^{n} \cdot \mathcal{O}_{F}$.

(3) For every $k \geq 1$, we have $\partial^{k} \mathcal{O}_{F}=\mathbb{T}_{k}^{n} \cdot \mathcal{O}_{F} \backslash \mathbb{T}_{<k}^{n} \cdot \mathcal{O}_{F}$.

(4) We have $\bigcup_{i=0}^{m} \mathbb{T}^{n} \cdot f_{i}=\bigcup_{j=0}^{\infty} \partial^{j} \mathcal{O}_{F}$, where the right-hand side is a disjoint union.

(5) Any $F$-term $t f_{i} \in \mathbb{T}^{n} \cdot f_{i} \backslash \mathcal{O}_{F}$ is divisible by an F-term in $\partial \mathcal{O}_{F}$.

Proof. First we show (1) by induction on $k$. For $k=0$, the claim follows from the definition. For $k=1$, we have $\overline{\partial^{1} \mathcal{O}_{F}}=\partial^{0} \mathcal{O}_{F} \cup \partial^{1} \mathcal{O}_{F}$ by Definition 3.1. Inductively, it follows that $\overline{\partial^{k+1} \mathcal{O}_{F}}=\overline{\partial^{k} \mathcal{O}_{F}} \cup \partial^{k+1} \mathcal{O}_{F}=\bigcup_{i=0}^{k+1} \partial^{i} \mathcal{O}_{F}$. This is a disjoint union, since $\partial^{k+1} \mathcal{O}_{F} \cap \overline{\partial^{k} \mathcal{O}_{F}}=\emptyset$ in each step.

Next, we prove claim (2). Again we proceed by induction on $k$, the case $k=0$ being obviously true. Inductively, we have $\overline{\partial^{k+1} \mathcal{O}_{F}}=\overline{\partial^{k} \mathcal{O}_{F}} \cup \partial^{k+1} \mathcal{O}_{F}=\mathbb{T}_{\leq k}^{n}$. $\mathcal{O}_{F} \cup \mathbb{T}_{1}^{n} \cdot\left(\mathbb{T}_{\leq k}^{n} \cdot \mathcal{O}_{F}\right)=\mathbb{T}_{\leq k+1}^{n} \cdot \mathcal{O}_{F}$

Claim (3) is a consequence of (2) and the equality $\partial^{k} \mathcal{O}_{F}=\overline{\partial^{k} \mathcal{O}_{F}} \backslash \overline{\partial^{k-1} \mathcal{O}_{F}}$.

Inclusion " $\subseteq$ " in claim (4) follows from the observation that, by (2), every $F$-term is in $\overline{\partial^{k} \mathcal{O}_{F}}$ for some $k \geq 0$. The converse inclusion holds by (3).

Finally, claim (5) holds because (4) implies that $t f_{i} \in \partial^{k} \mathcal{O}_{F}$ for some $k \geq 1$, and by (3) this is equivalent to the existence of a factorization $t=t^{\prime} t^{\prime \prime}$ where $\operatorname{deg}\left(t^{\prime}\right)=k-1$ and $t^{\prime \prime} f_{i} \in \partial \mathcal{O}_{F}$.

In view of this result, the following definition appears natural.

Definition 3.3. Let $F=\left\{f_{1}, \ldots, f_{m}\right\} \subset P \backslash\{0\}$, and let $\mathcal{O}_{F}$ be an $F$-order ideal.

(1) For an $F$-term $t f_{i} \in \mathcal{O}_{F}$, we define $\operatorname{ind}_{\mathcal{O}_{F}}\left(t f_{i}\right)=\min \left\{k \geq 0 \mid t f_{i} \in \overline{\partial^{k} \mathcal{O}_{F}}\right\}$ and call it the $\mathcal{O}_{F}$-index of $t f_{i}$.

(2) Given a non-zero polynomial $f \in J$, we write $f=p_{1} f_{1}+\cdots+p_{m} f_{m}$ with $p_{i} \in P$ and we let $\mathcal{P}=\left(p_{1} f_{1}, \ldots, p_{m} f_{m}\right)$. Then the number

$$
\operatorname{ind}_{\mathcal{O}_{F}}(\mathcal{P})=\max \left\{\operatorname{ind}_{\mathcal{O}_{F}}\left(t f_{i}\right) \mid i \in\{1, \ldots, m\}, t \in \operatorname{Supp}\left(p_{i}\right)\right\}
$$

is called the $\mathcal{O}_{F}$-index of the representation $\mathcal{P}$ of $f$.

In other words, the $\mathcal{O}_{F}$-index of $t f_{i}$ is the unique number $k \geq 0$ such that $t f_{i} \in \partial^{k} \mathcal{O}_{F}$. Note that the $\mathcal{O}_{F}$-index of a polynomial $f \in J$ depends on the representation of $f$ in terms of the generators of $J$. It is not clear how to find a representation $\mathcal{P}$ which yields the smallest ind $\mathcal{O}_{F}(\mathcal{P})$. Using the Subideal Border Division Algorithm, we shall address this point below.

The following proposition collects some basic properties of the $\mathcal{O}_{F}$-index.

Proposition 3.4. Let $F=\left\{f_{1}, \ldots, f_{m}\right\} \subset P \backslash\{0\}$, and let $\mathcal{O}_{F}$ be an $F$-order ideal.

(1) For an $F$-term $t f_{i} \in \mathbb{T}^{n} \cdot f_{i}$, the number $k=\operatorname{ind}_{\mathcal{O}_{F}}\left(t f_{i}\right)$ is the smallest natural number such that there exists a factorization $t=t^{\prime} t^{\prime \prime}$ with a term $t^{\prime} \in \mathbb{T}^{n}$ of degree $k$ and with $t^{\prime \prime} f_{i} \in \mathcal{O}_{F}$.

(2) Given $t \in \mathbb{T}^{n}$ and an $F$-term $t^{\prime} f_{i} \in \mathbb{T}^{n} \cdot f_{i}$, we have

$$
\operatorname{ind}_{\mathcal{O}_{F}}\left(t t^{\prime} f_{i}\right) \leq \operatorname{deg}(t)+\operatorname{ind}_{\mathcal{O}_{F}}\left(t^{\prime} f_{i}\right) \text {. }
$$


(3) For $f, g \in J \backslash\{0\}$ such that $f+g \neq 0$, we write $f=p_{1} f_{1}+\cdots+p_{m} f_{m}$ and $g=q_{1} f_{1}+\cdots+q_{m} f_{m}$ with $p_{i}, q_{j} \in P$, and we let $\mathcal{P}=\left(p_{1} f_{1}, \ldots, p_{m} f_{m}\right)$ and $\mathcal{Q}=\left(q_{1} f_{1}, \ldots, q_{m} f_{m}\right)$. Then we have

$$
\operatorname{ind}_{\mathcal{O}_{F}}(\mathcal{P}+\mathcal{Q}) \leq \max \left\{\operatorname{ind}_{\mathcal{O}_{F}}(\mathcal{P}), \operatorname{ind}_{\mathcal{O}_{F}}(\mathcal{Q})\right\}
$$

(4) Given $f \in J \backslash\{0\}$, we write $f=p_{1} f_{1}+\cdots+p_{m} f_{m}$ with $p_{i} \in P$ and let $\mathcal{P}=\left(p_{1} f_{1}, \ldots, p_{m} f_{m}\right)$. For every $g \in P \backslash\{0\}$, we then have

$$
\operatorname{ind}_{\mathcal{O}_{F}}(g \mathcal{P}) \leq \operatorname{deg}(g)+\operatorname{ind}_{\mathcal{O}_{F}}(\mathcal{P}) .
$$

Proof. The first claim follows from Proposition 3.2 The second claim follows from the first. The third claim is a consequence of the fact that every $F$-term appearing in $\mathcal{P}+\mathcal{Q}$ appears in $\mathcal{P}$ or $\mathcal{Q}$. The last claim follows from (2) and the observation that $g \mathcal{P}$ is a $K$-linear combination of tuples $t \mathcal{P}$ with $t \in \operatorname{Supp}(g)$.

Now we have collected enough material to formulate and prove the subideal version of the Border Division Algorithm.

Algorithm 3.5 (The Subideal Border Division Algorithm). Let $F=\left\{f_{1}, \ldots, f_{m}\right\} \subset$ $P \backslash\{0\}$, let $J=\left\langle f_{1}, \ldots, f_{m}\right\rangle$, let $\mathcal{O}_{F}=\left\{t_{1} f_{\alpha_{1}}, \ldots, t_{\mu} f_{\alpha_{\mu}}\right\}$ be an $F$-order ideal where $\alpha_{i} \in\{1, \ldots, m\}, t_{i} \in \mathcal{O}_{\alpha_{i}}$, let $\partial \mathcal{O}_{F}=\left\{b_{1} f_{\beta_{1}}, \ldots, b_{\nu} f_{\beta_{\nu}}\right\}$ be its border, and let $G=\left(g_{1}, \ldots, g_{\nu}\right)$ be an $\mathcal{O}_{F}$-subideal border prebasis, where $g_{j}=b_{j} f_{\beta_{j}}-\sum_{i=1}^{\mu} c_{i j} t_{i} f_{\alpha_{i}}$ with $c_{1 j}, \ldots, c_{\mu j} \in K$ for $j=1, \ldots, \nu$. Given a polynomial $f \in J$, we write $f=$ $p_{1} f_{1}+\cdots+p_{m} f_{m}$ and consider the following instructions.

D1 Let $h_{1}=\cdots=h_{\nu}=0, c_{1}=\cdots=c_{\mu}=0$, and $\mathcal{Q}=\left(q_{1} f_{1}, \ldots, q_{m} f_{m}\right)$ with $q_{i}=p_{i}$ for $i=1, \ldots, m$.

D2 Repeat the following steps until $\mathcal{Q}=(0, \ldots, 0)$. Then return the tuple $\left(h_{1}, \ldots, h_{\nu}, c_{1}, \ldots, c_{\mu}\right)$ and stop.

D3 Repeat the following step until $\operatorname{ind}_{\mathcal{O}_{F}}(\mathcal{Q})=0$. Then find $c_{1}, \ldots, c_{\mu} \in K$ such that $q_{1} f_{1}+\cdots+q_{m} f_{m}=c_{1} t_{1} f_{\alpha_{1}}+\cdots+c_{\mu} t_{\mu} f_{\alpha_{\mu}}$. Return the tuple $\left(h_{1}, \ldots, h_{\nu}, c_{1}, \ldots, c_{\mu}\right)$ and stop.

D4 If $\operatorname{ind}_{\mathcal{O}_{F}}(\mathcal{Q})>0$, then determine the smallest index $i \in\{1, \ldots, m\}$ such that there exists a term $t \in \operatorname{Supp}\left(q_{i}\right)$ with $\operatorname{ind}_{\mathcal{O}_{F}}\left(t f_{i}\right)=\operatorname{ind}_{\mathcal{O}_{F}}(\mathcal{Q})$. Choose such a term $t$. Let $a \in K$ be the coefficient of $t$ in $q_{i}$. Next, determine the smallest index $j \in\{1, \ldots, \nu\}$ such that $t$ factors as $t=t^{\prime} t^{\prime \prime}$ with a term $t^{\prime}$ of degree $\operatorname{ind}_{\mathcal{O}_{F}}\left(t f_{i}\right)-1$ and with $t^{\prime \prime} f_{i}=b_{j} f_{\beta_{j}} \in \partial \mathcal{O}_{F}$. Subtract the tuple corresponding to the representation

$$
a t^{\prime} g_{j}=a t^{\prime} b_{j} f_{\beta_{j}}-\sum_{i=1}^{\mu} c_{i j} a t^{\prime} t_{i} f_{\alpha_{i}}
$$

from $\mathcal{Q}$, add at' to $h_{j}$, and continue.

This is an algorithm which returns a tuple $\left(h_{1}, \ldots, h_{\nu}, c_{1}, \ldots, c_{\mu}\right) \in P^{\nu} \times K^{\mu}$ such that

$$
f=h_{1} g_{1}+\cdots+h_{\nu} g_{\nu}+c_{1} t_{1} f_{\alpha_{1}}+\cdots+c_{\mu} t_{\mu} f_{\alpha_{\mu}}
$$

and $\operatorname{deg}\left(h_{i}\right) \leq \operatorname{ind}_{\mathcal{O}_{F}}(\mathcal{P})-1$ for $\mathcal{P}=\left(p_{1} f_{1}, \ldots, p_{m} f_{m}\right)$ and for all $i \in\{1, \ldots, \nu\}$ with $h_{i} \neq 0$. This representation does not depend on the choice of the term $t$ in step $\mathbf{D} 4$.

Proof. First we show that all of the steps can be executed. In step D3, the condition $\operatorname{ind}_{\mathcal{O}_{F}}(\mathcal{Q})=0$ implies that all $F$-terms $t f_{i}$ with $t \in \operatorname{Supp}\left(q_{i}\right)$ are contained in $\mathcal{O}_{F}$. In step D4, the definition of $\operatorname{ind}_{\mathcal{O}_{F}}(\mathcal{Q})$ implies that a term $t$ of the desired kind 
exists. By Proposition 3.4 1, this term $t$ has a factorization $t=t^{\prime} t^{\prime \prime}$ with the desired properties.

Next, we prove termination by showing that step D4 is performed only finitely many times. Let us investigate the subtraction of the representation of $a t^{\prime} g_{j}$ from $\mathcal{Q}$. By the choice of $t^{\prime}$, the $\mathcal{O}_{F}$-index of $t^{\prime} b_{j} f_{\beta_{j}}$ is $\operatorname{deg}\left(t^{\prime}\right)$ more than the $\mathcal{O}_{F}$-index of $b_{j} f_{\beta_{j}}$. By Proposition 3.4, 2, this is the maximal increase, and the $\mathcal{O}_{F}$-index of the other $F$-terms in the representation of $a t^{\prime} g_{j}$ is smaller than $\operatorname{ind}_{\mathcal{O}_{F}}(\mathcal{Q})$. Thus the number of $F$-terms in $\mathcal{Q}$ of maximal $\mathcal{O}_{F}$-index decreases by the subtraction, and after finitely many steps the algorithm reaches step D2 or D3 and stops.

Finally, we prove correctness. To do so, we show that the equality

$$
f=q_{1} f_{1}+\cdots+q_{m} f_{m}+h_{1} g_{1}+\cdots+h_{\nu} g_{\nu}+c_{1} t_{1} f_{\alpha_{1}}+\cdots+c_{\mu} t_{\mu} f_{\alpha_{\mu}}
$$

is an invariant of the algorithm. It is satisfied at the end of step D1. The constants $c_{1}, \ldots, c_{\mu}$ are only changed in step D3. In this case the contribution $q_{1} f_{1}+\cdots+$ $q_{m} f_{m}$ to the above equality is replaced by the equal contribution $c_{1} t_{1} f_{\alpha_{1}}+\cdots+$ $c_{\mu} t_{\mu} f_{\alpha_{\mu}}$. The tuple $\mathcal{Q}$ is only changed in step $\mathbf{D} 4$. There the subtraction of the representation of $a t^{\prime} g_{j}$ from $\mathcal{Q}$ and the corresponding change in $q_{1} f_{1}+\cdots+q_{m} f_{m}$ are compensated by the addition of $a t^{\prime}$ to $h_{j}$ and the corresponding change in $h_{1} g_{1}+\cdots+h_{\nu} g_{\nu}$. When the algorithm stops, we have $q_{1}=\cdots=q_{m}=0$. This proves the claimed representation of $f$. Moreover, only terms $t^{\prime}$ of degree $\operatorname{deg}\left(t^{\prime}\right) \leq$ $\operatorname{ind}_{\mathcal{O}_{F}}(\mathcal{Q})-1 \leq \operatorname{ind}_{\mathcal{O}_{F}}(\mathcal{P})-1$ are added to $h_{j}$

The additional claim that the result of the algorithm does not depend on the choice of $t$ in step $\mathbf{D} 4$ follows from the observation that $t f_{i}$ is replaced by $F$-terms of strictly smaller $\mathcal{O}_{F}$-index. Thus the different executions of step $\mathbf{D} 4$ corresponding to the reduction of several $F$-terms of maximal $\mathcal{O}_{F}$-index in $\mathcal{Q}$ do not interfere with one another, and the final result, after all of those $F$-terms have been rewritten, is independent of the order in which they are taken care of.

Now we come back to the observation made above that it is not clear how to find a representation $\mathcal{P}$ having the smallest $\operatorname{ind}_{\mathcal{O}_{F}}(\mathcal{P})$. First of all, notice that in step D4 the algorithm uses a term $t$ which is not uniquely determined. Also, there may be several factorizations of $t$. We choose the indices $i$ and $j$ minimally to determine this step of the algorithm uniquely, but this particular choice is not forced upon us. Moreover, it is clear that the result of the division depends on the numbering of the elements of $\partial \mathcal{O}_{F}$.

As indicated above, the Subideal Border Division Algorithm has important implications. The following corollaries comprise a few of them.

Corollary 3.6 (Subideal Border Bases and Special Generation). In the setting of the algorithm, let $I=\langle G\rangle$. Then the tuple $G$ is an $\mathcal{O}_{F}$-subideal border basis of $I$ if and only if one of the following equivalent conditions is satisfied.

$\left(A_{1}\right)$ For every non-zero polynomial $f \in I \cap J$ with a representation $f=p_{1} f_{1}+$ $\cdots+p_{m} f_{m}$ and $\mathcal{P}=\left(p_{1} f_{1}, \ldots, p_{m} f_{m}\right)$, there exist polynomials $h_{1}, \ldots, h_{\nu} \in$ $P$ such that $f=h_{1} g_{1}+\cdots+h_{\nu} g_{\nu}$ and $\operatorname{deg}\left(h_{i}\right) \leq \operatorname{ind}_{\mathcal{O}_{F}}(\mathcal{P})-1$ whenever $h_{i} g_{i} \neq 0$.

$\left(A_{2}\right)$ For every non-zero polynomial $f \in I \cap J$ with a representation $f=p_{1} f_{1}+$ $\cdots+p_{m} f_{m}$ and $\mathcal{P}=\left(p_{1} f_{1}, \ldots, p_{m} f_{m}\right)$, there exist $h_{1}, \ldots, h_{\nu} \in P$ such that $f=h_{1} g_{1}+\cdots+h_{\nu} g_{\nu}$ and $\max \left\{\operatorname{deg}\left(h_{i}\right) \mid i \in\{1, \ldots, \nu\}, h_{i} g_{i} \neq 0\right\}=$ $\operatorname{ind}_{\mathcal{O}_{F}}(\mathcal{P})-1$. 
Proof. First we show that $\left(A_{1}\right)$ holds if $G$ is an $\mathcal{O}_{F}$-border basis. The Subideal Border Division Algorithm computes a representation $f=h_{1} g_{1}+\cdots+h_{\nu} g_{\nu}+$ $c_{1} t_{1} f_{\alpha_{1}}+\cdots+c_{\mu} t_{\mu} f_{\alpha_{\mu}}$ with $h_{1}, \ldots, h_{\nu} \in P$ and $c_{1}, \ldots, c_{\mu} \in K$ such that $\operatorname{deg}\left(h_{i}\right) \leq$ $\operatorname{ind}_{\mathcal{O}_{F}}(\mathcal{P})-1$ for $i=1, \ldots, \nu$. Then $c_{1} t_{1} f_{\alpha_{1}}+\cdots+c_{\mu} t_{\mu} f_{\alpha_{\mu}} \equiv 0$ modulo $I \cap J$, and the hypothesis implies $c_{1}=\cdots=c_{\mu}=0$.

Next, we prove that $\left(A_{1}\right)$ implies $\left(A_{2}\right)$. If $\operatorname{deg}\left(h_{i}\right)<\operatorname{ind}_{\mathcal{O}_{F}}(\mathcal{P})-1$, then Proposition 3.4.2 shows that the $\mathcal{O}_{F}$-index of every representation of $h_{i} g_{i}$ is at most $\operatorname{deg}\left(h_{i}\right)+1$ and hence smaller than $\operatorname{ind}_{\mathcal{O}_{F}}(\mathcal{P})$. By Proposition 3.4 4, there has to be at least one number $i \in\{1, \ldots, \nu\}$ such that $\operatorname{deg}\left(h_{i}\right)=\operatorname{ind}_{\mathcal{O}_{F}}(\mathcal{P})-1$.

Finally, we assume $\left(A_{2}\right)$ and show the subideal border basis property. Let $c_{1}, \ldots, c_{\mu} \in K$ satisfy $c_{1} t_{1} f_{\alpha_{1}}+\cdots+c_{\mu} t_{\mu} f_{\alpha_{\mu}} \in I \cap J$. Then either $f=c_{1} t_{1} f_{\alpha_{1}}+$ $\cdots+c_{\mu} t_{\mu} f_{\alpha_{\mu}}$ equals the zero polynomial or not. In the latter case we apply $\left(A_{2}\right)$ and obtain a representation $f=h_{1} g_{1}+\cdots+h_{\nu} g_{\nu}$ with $h_{1}, \ldots, h_{\nu} \in P$. Since $f \neq 0$, we have $\max \left\{\operatorname{deg}\left(h_{i}\right) \mid i \in\{1, \ldots, \nu\}, h_{i} g_{i} \neq 0\right\} \geq 0$. $\operatorname{But}_{\operatorname{ind}_{\mathcal{O}_{F}}}(\mathcal{P})-1=-1$ is in contradiction to the second part of $\left(A_{2}\right)$. Hence we must have $f=0$. Thus $I \cap J \cap\left\langle\mathcal{O}_{F}\right\rangle_{K}=0$, i.e., the tuple $G$ is an $\mathcal{O}_{F}$-subideal border basis of $I$.

Definition 3.7. In the setting of the algorithm, the polynomial

$$
\mathrm{NR}_{\mathcal{O}_{F}, G}(\mathcal{P})=c_{1} t_{1} f_{\alpha_{1}}+\cdots+c_{\mu} t_{\mu} f_{\alpha_{\mu}}
$$

is called the normal remainder of the representation $\mathcal{P}=\left(p_{1} f_{1}, \ldots, p_{m} f_{m}\right)$ of $f$ with respect to $G$.

Clearly, the normal remainder depends on the choice of the representation $\mathcal{P}$. It has the following application.

Corollary 3.8. In the setting of the algorithm, the residue classes of the elements of $\mathcal{O}_{F}$ generate the image of the ideal $J$ in $P /\langle G\rangle$ as a $K$-vector space.

In other words, the residue class of every polynomial $f \in J$ can be represented as a $K$-linear combination of the residue classes $\left\{\bar{t}_{1} \bar{f}_{\alpha_{1}}, \ldots, \bar{t}_{\mu} \bar{f}_{\alpha_{\mu}}\right\}$. Indeed, such a representation can be found by computing the normal remainder $\mathrm{NR}_{\mathcal{O}_{F}, G}(\mathcal{P})$ of the representation $\mathcal{P}=\left(p_{1} f_{1}, \ldots, p_{m} f_{m}\right)$ of $f=p_{1} f_{1}+\cdots+p_{m} f_{m}$.

Proof. By the algorithm, every $f \in J$ can be represented in the form $f=h_{1} g_{1}+$ $\cdots+h_{\nu} g_{\nu}+c_{1} t_{1} f_{\alpha_{1}}+\cdots+c_{\mu} t_{\mu} f_{\alpha_{\mu}}$, where $h_{1}, \ldots, h_{\nu} \in P$ and $c_{1}, \ldots, c_{\mu} \in K$. Forming residue classes modulo $\langle G\rangle$ yields the claim.

The next corollary provides another motivation for the name "subideal border basis".

Corollary 3.9. In the setting of the algorithm, let $G$ be an $\mathcal{O}_{F}$-subideal border basis of an ideal $I \subset P$. Then $G$ generates the ideal $I \cap J$.

Proof. By definition, we have $\left\langle g_{1}, \ldots, g_{\nu}\right\rangle \subseteq I \cap J$. To prove the converse inclusion, let $f \in I \cap J$. Using the Subideal Border Division Algorithm, the polynomial $f$ can be expanded as $f=h_{1} g_{1}+\cdots+h_{\nu} g_{\nu}+c_{1} t_{1} f_{\alpha_{1}}+\cdots+c_{\mu} t_{\mu} f_{\alpha_{\mu}}$, where $h_{1}, \ldots, h_{\nu} \in P$ and $c_{1}, \ldots, c_{\mu} \in K$. This implies the equality of residue classes $0=\bar{f}=c_{1} \bar{t}_{1} \bar{f}_{\alpha_{1}}+$ $\cdots+c_{\mu} \bar{t}_{\mu} \bar{f}_{\alpha_{\mu}}$ in $P / I$. By assumption, the residue classes $\bar{t}_{1} \bar{f}_{\alpha_{1}}, \ldots, \bar{t}_{\mu} \bar{f}_{\alpha_{\mu}}$ form a $K$-vector space basis of $(I+J) / I$. Hence, $c_{1}=\cdots=c_{\mu}=0$, and the expansion of $f$ yields $f=h_{1} g_{1}+\cdots+h_{\nu} g_{\nu} \in\langle G\rangle$. 


\section{The SUBIDEAL VERSION OF THE BM-ALGORITHM}

Let $K$ be a field, let $P=K\left[x_{1}, \ldots, x_{n}\right]$ be the polynomial ring in $n$ indeterminates over $K$, equipped with the standard grading, and let $\mathbb{T}^{n}$ be the monoid of terms in $P$. Given a finite set of points $\mathbb{X}=\left\{p_{1}, \ldots, p_{s}\right\} \subseteq K^{n}$, we let eval $: P \longrightarrow K^{s}$ be the evaluation map eval $(f)=\left(f\left(p_{1}\right), \ldots, f\left(p_{s}\right)\right)$ associated to $\mathbb{X}$. It is easy to adjust the Buchberger-Möller Algorithm (BM-algorithm) so that it computes a border basis of the vanishing ideal

$$
I_{\mathbb{X}}=\left\langle f \in P \mid f\left(p_{1}\right)=\cdots=f\left(p_{s}\right)=0\right\rangle=\operatorname{ker}(\text { eval }) \subseteq P
$$

of $\mathbb{X}$. Since we use a version which differs slightly from the standard formulation (see for instance [4] or [10], Thm. 6.3.10), let us briefly recall its main steps.

Algorithm 4.1 (BM-Algorithm for Border Bases). Let $\mathbb{X}=\left\{p_{1}, \ldots, p_{s}\right\} \subseteq K^{n}$ be a set of points given by their coordinates, and let $\sigma$ be a degree compatible term ordering on $\mathbb{T}^{n}$. The following instructions define an algorithm which computes the order ideal $\mathcal{O}_{\sigma}(I)=\mathbb{T}^{n} \backslash \operatorname{LT}_{\sigma}\left(I_{\mathbb{X}}\right)$ and the $\mathcal{O}_{\sigma}\left(I_{\mathbb{X}}\right)$-border basis $G$ of $I_{\mathbb{X}}$.

B1 Let $d=1, \mathcal{O}=\{1\}, G=\emptyset$, and $\mathcal{M}=(1, \ldots, 1)^{\operatorname{tr}} \in \operatorname{Mat}_{s, 1}(K)$, and let $L=\left(t_{1}, \ldots, t_{\ell}\right)$ be the tuple of all terms of degree 1 , ordered decreasingly w.r.t. $\sigma$.

B2 Repeat the following steps until $L=\emptyset$. Then return $(\mathcal{O}, G)$ and stop.

B3 Form the matrix $\mathcal{A}=\left(\operatorname{eval}\left(t_{1}\right)|\cdots| \operatorname{eval}\left(t_{\ell}\right) \mid \mathcal{M}\right)$ of size $s \times\left(\ell+m^{\prime}\right)$ where $m^{\prime}$ is the number of columns of $\mathcal{M}$. Compute a matrix $\mathcal{B}$ whose rows are a basis of the kernel of $\mathcal{A}$. Let $k$ be the number of rows of $\mathcal{B}$.

B4 Reduce $\mathcal{B}$ to a matrix $\mathcal{C}=\left(c_{i j}\right) \in \operatorname{Mat}_{k, \ell+m^{\prime}}(K)$ in reduced row echelon form.

B5 For all $j \in\{1, \ldots, \ell\}$ such that there exists an $i \in\{1, \ldots, k\}$ with pivot index $\nu(i)=j$, append the polynomial

$$
t_{j}+\sum_{j^{\prime}=j+1}^{\ell} c_{i j^{\prime}} t_{j^{\prime}}+\sum_{j^{\prime}=\ell+1}^{\ell+m^{\prime}} c_{i j^{\prime}} u_{j^{\prime}}
$$

to the tuple $G$, where $u_{j^{\prime}}$ is the $\left(j^{\prime}-\ell\right)^{\text {th }}$ element of $\mathcal{O}$.

B6 For all $j=\ell, \ell-1, \ldots, 1$ such that the $j^{\text {th }}$ column of $\mathcal{C}$ contains no pivot element, append the term $t_{j}$ as a new first element to $\mathcal{O}$, append the column eval $\left(t_{j}\right)$ as a new first column to $\mathcal{M}$, increase $d$ by one, and let $L=$ $\left(t_{1}, \ldots, t_{\ell}\right)$ be the tuple of all terms of degree $d$ in $\partial \mathcal{O}$, ordered decreasingly w.r.t. $\sigma$.

The proof of this modified version is simply obtained by combining all the iterations of the usual BM-algorithm corresponding to terms of degree $d$ into one "block". The fact that we put the terms of degree $d$ in $\partial \mathcal{O}$ into $L$ in step B6 effects the computation of the entire border basis, rather than just the reduced $\sigma$-Gröbner basis of $I_{\mathbb{X}}$ (see [5], Thm. 3.3). A further elaboration is beyond the scope of the present paper and is left to the interested reader.

Given $\mathbb{X}$ and the polynomial ideal $J=\langle F\rangle$ with $F=\left\{f_{1}, \ldots, f_{m}\right\} \subset P \backslash\{0\}$, we know that the vanishing ideal $I_{\mathbb{X}}$ has an $\mathcal{O}_{\sigma}(I)_{F}$-subideal border basis. The following generalization of the BM-algorithm computes this subideal border basis. 
Algorithm 4.2 (Subideal Version of the BM-Algorithm). Let $\mathbb{X}=\left\{p_{1}, \ldots, p_{s}\right\} \subseteq$ $K^{n}$ be a set of points given by their coordinates, let $\sigma$ be a degree compatible term ordering, and let $F=\left\{f_{1}, \ldots, f_{m}\right\} \subset P \backslash\{0\}$ be a set of polynomials which generate an ideal $J=\langle F\rangle$. The following instructions define an algorithm which computes an $F$-order ideal $\mathcal{O}_{\sigma}(I)_{F}$ and the $\mathcal{O}_{\sigma}(I)_{F}$-subideal border basis $G$ of $I_{\mathbb{X}}$.

S1 Let $d=\min \left\{\operatorname{deg}\left(f_{1}\right), \ldots, \operatorname{deg}\left(f_{m}\right)\right\}, \mathcal{O}_{F}=\emptyset, G=\emptyset$, and $\mathcal{M} \in \operatorname{Mat}_{s, 0}(K)$. Let $L=\left(t_{1} f_{\alpha_{1}}, \ldots, t_{\ell} f_{\alpha_{\ell}}\right)$ be the tuple of all $F$-terms of degree $d$ in $F \cup \partial \mathcal{O}_{F}$, with their leading terms ordered decreasingly w.r.t. $\sigma$.

S2 Repeat the following steps until $L=\emptyset$ and $d \geq \max \left\{\operatorname{deg}\left(f_{1}\right), \ldots, \operatorname{deg}\left(f_{m}\right)\right\}$. Then return $\left(\mathcal{O}_{F}, G\right)$ and stop.

S3 Form the matrix $\mathcal{A}=\left(\operatorname{eval}\left(t_{1} f_{\alpha_{1}}\right)|\cdots| \operatorname{eval}\left(t_{\ell} f_{\alpha_{\ell}}\right) \mid \mathcal{M}\right)$ of size $s \times\left(\ell+m^{\prime}\right)$ where $m^{\prime}$ is the number of columns of $\mathcal{M}$. Compute a matrix $\mathcal{B}$ whose rows are a basis of the kernel of $\mathcal{A}$. Let $k$ be the number of rows of $\mathcal{B}$.

$\mathbf{S} 4$ Reduce $\mathcal{B}$ to a matrix $\mathcal{C}=\left(c_{i j}\right) \in \operatorname{Mat}_{k, \ell+m^{\prime}}(K)$ in reduced row echelon form.

S5 For all $j \in\{1, \ldots, \ell\}$ such that there exists an $i \in\{1, \ldots, k\}$ with pivot index $\nu(i)=j$, append the polynomial

$$
t_{j} f_{\alpha_{j}}+\sum_{j^{\prime}=j+1}^{\ell} c_{i j^{\prime}} t_{j^{\prime}} f_{\alpha_{j^{\prime}}}+\sum_{j^{\prime}=\ell+1}^{\ell+m^{\prime}} c_{i j^{\prime}} u_{j^{\prime}}
$$

to the tuple $G$, where $u_{j^{\prime}}$ is the $\left(j^{\prime}-\ell\right)^{\text {th }}$ element of $\mathcal{O}_{F}$.

S6 For all $j=\ell, \ell-1, \ldots, 1$ such that the $j^{\text {th }}$ column of $\mathcal{C}$ contains no pivot element, append the $F$-term $t_{j} f_{\alpha_{j}}$ as a new first element to $\mathcal{O}_{F}$, and append the column eval $\left(t_{j} f_{\alpha_{j}}\right)$ as a new first column to $\mathcal{M}$. Increase $d$ by one, let $L=\left(t_{1} f_{\alpha_{1}}, \ldots, t_{\ell} f_{\alpha_{\ell}}\right)$ be the tuple of all $F$-terms of degree $d$ in $F \cup \partial \mathcal{O}_{F}$, with their leading terms ordered decreasingly w.r.t. $\sigma$, and continue.

Proof. First we show finiteness. When a new degree is started in step $\mathbf{S 1}$ or S6, the matrix $\mathcal{M}$ has $m=\# \mathcal{O}_{F}$ columns where $\mathcal{O}_{F}$ is the current list of $F$-terms. In step $\mathbf{S 6}$ we enlarge $\mathcal{M}$ by new first columns which are linearly independent of the other columns. This can happen only finitely many times. Eventually we arrive at a situation where all new columns eval $\left(t_{i} f_{\alpha_{i}}\right)$ of $\mathcal{A}$ in step $\mathbf{S 3}$ are linearly dependent on the previous columns, and therefore the corresponding column of $\mathcal{C}$ contains a pivot element. Consequently, no elements are appended to $\mathcal{O}_{F}$ in that degree and we get $L=\emptyset$ in the next degree. Hence the algorithm stops.

Now we show correctness. The columns of $\mathcal{A}$ are the evaluation vectors of $F$ terms whose leading terms are ordered decreasingly w.r.t. $\sigma$. A row $\left(c_{i 1}, \ldots, c_{i \ell+m^{\prime}}\right)$ of $\mathcal{C}$ corresponds to a linear combination of these $F$-terms whose evaluation vector is zero. Let $g_{1}, \ldots, g_{k}$ be the polynomials given by these linear combinations of $F$-terms. Clearly, we have $g_{i} \in I_{\mathbb{X}} \cap J$.

The evaluation vectors of the $F$-terms which are put into $\mathcal{O}_{F}$ in step $\mathbf{S 6}$ are linearly independent of the evaluation vectors of the $F$-terms in the previous set $\mathcal{O}_{F}$ since there is no linear relation leading to a pivot element in the corresponding column of $\mathcal{C}$. Inductively, it follows that the evaluation vectors of the $F$-terms in $\mathcal{O}_{F}$ are always linearly independent. Henceforth, the pivot elements of $\mathcal{C}$ are always in the "new" columns and the polynomials $g_{i}$ have degree $d$. By the way the algorithm proceeds, every $F$-term in the border of the final set $\mathcal{O}_{F}$ appears in 
exactly one on the elements of $G$. All the other summands of a polynomial $g_{i}$ are in $\mathcal{O}_{F}$. Hence the final tuple $G$ is an $\mathcal{O}_{F}$-subideal border prebasis.

Furthermore, every $F$-term is either in $\mathcal{O}_{F}$ or it is a multiple of an $F$-term in $\partial \mathcal{O}_{F}$ (cf. Proposition 3.4,5). In the latter case, its evaluation vector can be written as a linear combination of the evaluation vectors of the elements of $\mathcal{O}_{F}$. Thus the evaluation vectors of the elements of $\mathcal{O}_{F}$ generate the space of all evaluation vectors of $F$-terms. Since they are linearly independent, they form a $K$-basis of that space. Now we use the facts that evaluation yields an isomorphism of $K$-vector spaces eval $: P / I \longrightarrow K^{s}$ and that the residue classes of the $F$-terms generate the $K$-vector subspace $(I+J) / I$ of $P / I$ to conclude that the residue classes of the $F$-terms in the final set $\mathcal{O}_{F}$ form a $K$-basis of $(I+J) / I$.

Let us illustrate this algorithm by an example.

Example 4.3. In the polynomial ring $P=\mathbb{Q}[x, y, z]$, we consider the ideal $J=\langle F\rangle$ with $F=\left\{f_{1}, f_{2}\right\}$ given by $f_{1}=x^{2}-1$ and $f_{2}=y-z$. Let $\sigma=$ DegRevLex.

We want to compute an $\mathcal{O}_{F}$-subideal border basis of the vanishing ideal of the point set $\mathbb{X}=\{(1,1,1),(0,1,1),(1,1,0),(1,0,1)\}$. Notice that the first point of $\mathbb{X}$ lies on $\mathcal{Z}\left(f_{1}, f_{2}\right)$, so that we should expect an $F$-order ideal consisting of three $F$ terms. Let us follow the steps of the algorithm. (We only list those steps in which something happens.)

S1 Let $d=1$ and $L=(y-z)$.

S3 Form $\mathcal{A}=(0,0,1,-1)^{\text {tr }}$ and compute $\mathcal{B}=(0)$. (Thus $\mathcal{C}=\mathcal{B}$.)

S6 Let $\mathcal{O}_{F}=\{y-z\}$ and $\mathcal{M}=(0,0,1,-1)^{\text {tr }}$. Let $d=2$ and $L=\left(x^{2}-1\right.$, $x(y-z), y(y-z), z(y-z))$.

S3 Compute $\mathcal{A}=\left(\begin{array}{ccccc}0 & 0 & 0 & 0 & 0 \\ -1 & 0 & 0 & 0 & 0 \\ 0 & 1 & 1 & 0 & 1 \\ 0 & -1 & 0 & -1 & -1\end{array}\right)$ and $\mathcal{B}=\left(\begin{array}{ccccc}0 & 1 & 0 & 0 & -1 \\ 0 & 0 & 1 & 1 & -1\end{array}\right)$. (Thus $\mathcal{C}=\mathcal{B}$.)

S5 The pivot indices $\nu(1)=2$ and $\nu(2)=3$ yield the tuple $G=\left(g_{1}, g_{2}\right)$ with $g_{1}=x(y-z)-(y-z)$ and $g_{2}=y(y-z)+z(y-z)-(y-z)$.

S6 We obtain $\mathcal{O}_{F}=\left\{x^{2}-1, z(y-z), y-z\right\}$ and $\mathcal{M}=\left(\begin{array}{rrr}0 & 0 & 0 \\ -1 & 0 & 0 \\ 0 & 0 & 1 \\ 0 & -1 & -1\end{array}\right)$.

Let $d=3$. We have $L=\left(x\left(x^{2}-1\right), y\left(x^{2}-1\right), z\left(x^{2}-1\right), x z(y-z), y z(y-z)\right.$, $\left.z^{2}(y-z)\right)$.

S3 Find $\mathcal{A}=\left(\begin{array}{rrrrrrrrr}0 & 0 & 0 & 0 & 0 & 0 & 0 & 0 & 0 \\ 0 & 0 & 0 & 0 & 0 & 0 & -1 & 0 & 0 \\ 0 & 0 & 0 & 0 & 0 & 0 & 0 & 0 & 1 \\ 0 & 0 & 0 & -1 & 0 & -1 & 0 & -1 & -1\end{array}\right)$ and $\mathcal{B}=\left(\begin{array}{rrrrrrrrr}1 & 0 & 0 & 0 & 0 & 0 & 0 & 0 & 0 \\ 0 & 1 & 0 & 0 & 0 & 0 & 0 & 0 & 0 \\ 0 & 0 & 1 & 0 & 0 & 0 & 0 & 0 & 0 \\ 0 & 0 & 0 & 1 & 0 & 0 & 0 & -1 & 0 \\ 0 & 0 & 0 & 0 & 1 & 0 & 0 & 0 & 0 \\ 0 & 0 & 0 & 0 & 0 & 1 & 0 & -1 & 0\end{array}\right)$. (Thus $\mathcal{C}=\mathcal{B}$.) 
S5 Here we obtain $G=\left(g_{1}, \ldots, g_{8}\right)$ where $g_{3}=x\left(x^{2}-1\right), g_{4}=y\left(x^{2}-1\right)$, $g_{5}=z\left(x^{2}-1\right), g_{6}=x z(y-z)-z(y-z), g_{7}=y z(y-z)$, and finally $g_{8}=z^{2}(y-z)-z(y-z)$.

S6 There are no new non-pivot indices. Hence $\mathcal{O}$ and $\mathcal{M}$ are not changed.

S2 We get $L=\emptyset$ and the algorithm stops.

The result is the $F$-order ideal $\mathcal{O}_{F}=\left\{x^{2}-1, z(y-z), y-z\right\}$ and the $\mathcal{O}_{F}$-subideal border basis $G=\left(g_{1}, \ldots, g_{8}\right)$ of $I_{\mathbb{X}}$.

\section{The SUbideAl Version OF THE AVI-ALGORIthm}

From here on we work in the polynomial $\operatorname{ring} P=\mathbb{R}\left[x_{1}, \ldots, x_{n}\right]$ over the field of real numbers. We let $\mathbb{X}=\left\{p_{1}, \ldots, p_{s}\right\} \subset[-1,1]^{n} \subset \mathbb{R}^{n}$ be a finite set of points and $\varepsilon>\tau>0$ two threshold numbers. (The number $\varepsilon$ can be thought of as a measure for error tolerance of the input data points $\mathbb{X}$ and $\tau$ is used as a "minimum size" for acceptable leading coefficients of unitary polynomials.)

Let us point out the following notational convention we are using: the "usual" norm of a polynomial $f \in P$ is the Euclidean norm of its coefficient vector and is denoted by $\|f\|$. By "unitary" we mean $\|f\|=1$. In contrast, by $\|f\|_{1}$ we mean the sum of the absolute values of the coefficients of $f$, and the term " \|\|$_{1}$-unitary" is to be interpreted accordingly.

Recall that we say that a polynomial $f \in P$ vanishes $\varepsilon$-approximately at a point $p \in \mathbb{R}^{n}$ if $|f(p)|<\varepsilon$. Furthermore, by eval $: P \longrightarrow \mathbb{R}^{s}$ we denote the evaluation map eval $(f)=\left(f\left(p_{1}\right), \ldots, f\left(p_{s}\right)\right)$ associated to $\mathbb{X}$. For the convenience of the reader, we briefly recall the basic structure of the Approximate Vanishing Ideal Algorithm (AVI-algorithm) from [5]. Notice that we skip several technical details and explicit error estimates. The goal of the AVI-algorithm is to compute an approximate border basis, a notion that is defined as follows.

Definition 5.1. Let $\mathcal{O}=\left\{t_{1}, \ldots, t_{\mu}\right\} \subseteq \mathbb{T}^{n}$ be an order ideal of terms, let $\partial \mathcal{O}=$ $\left\{b_{1}, \ldots, b_{\nu}\right\}$ be its border, and let $G=\left\{g_{1}, \ldots, g_{\nu}\right\}$ be an $\mathcal{O}$-border prebasis of the ideal $I=\left\langle g_{1}, \ldots, g_{\nu}\right\rangle$ in $P$. Recall that this means that $g_{j}$ is of the form $g_{j}=b_{j}-\sum_{i=1}^{\mu} c_{i j} t_{i}$ with $c_{i j} \in \mathbb{R}$.

For every pair $(i, j)$ such that $b_{i}, b_{j}$ are neighbors in $\partial \mathcal{O}$, we compute the normal remainder $S_{i j}^{\prime}=\mathrm{NR}_{\mathcal{O}, G}\left(S_{i j}\right)$ of the S-polynomial of $g_{i}$ and $g_{j}$ with respect to $G$. We say that $G$ is an $\varepsilon$-approximate border basis of the ideal $I=\langle G\rangle$ if we have $\left\|S_{i j}\right\|<\varepsilon$ for all such pairs $(i, j)$.

Moreover, the AVI-algorithm uses the concepts of approximate kernel and stabilized reduced row echelon form which were introduced in [5], Sect. 2 and 3. For the convenience of the reader, we recall these concepts.

Let $\mathcal{A} \in \operatorname{Mat}_{m, n}(\mathbb{R})$, and let $\mathcal{A}=\mathcal{U} \cdot\left(\begin{array}{ll}\mathcal{D} & 0 \\ 0 & 0\end{array}\right) \cdot \mathcal{V}^{\text {tr }}$ be the SVD of $\mathcal{A}$, where $\mathcal{D}=\operatorname{diag}\left(s_{1}, \ldots, s_{r}\right)$ is a diagonal matrix containing the singular values $s_{1} \geq s_{2} \geq$ $\cdots \geq s_{r}>0$. For $\varepsilon>0$, let $k \in\{1, \ldots, r\}$ be chosen such that $s_{k}>\varepsilon \geq s_{k+1}$. Form the matrix $\widetilde{\mathcal{A}}=\mathcal{U} \widetilde{\mathcal{S}} \mathcal{V}^{\text {tr }}$ by setting $s_{k+1}=\cdots=s_{r}=0$ in $\mathcal{S}$. It is called the $\varepsilon$-singular value truncation of $\mathcal{A}$. Then the vector subspace $\operatorname{apker}(\mathcal{A}, \varepsilon)=\operatorname{ker}(\widetilde{\mathcal{A}})$ is called the $\varepsilon$-approximate kernel of $\mathcal{A}$.

The computation of the stabilized reduced row echelon form of a matrix $A \in$ $\operatorname{Mat}_{m, n}(\mathbb{R})$ with respect to a given $\tau>0$ is a stabilized version of Gaussian reduction in the spirit of Shirayanagi and Sweedler (cf. [12]). For the precise formulation 
of its steps we refer to [5], Lemma 3.2. For us it is useful to recall the output of this algorithm: it computes a matrix $R$ in reduced row echelon form. The row space of $R$ is contained in the row space of the matrix $\bar{A}$ which is obtained from $A$ by setting the columns whose norm is less than $\tau$ to zero. Here the pivot elements of $R$ are not 1 , but its rows are unitary vectors. Furthermore, if the rows of $A$ are unitary and mutually orthogonal, the row vectors of $R$ differ by less than $\tau m \sqrt{n}$ from unitary vectors in the row space of $A$.

Now we are ready to recall the formulation of the AVI-algorithm.

Algorithm 5.2 (AVI-Algorithm). Let $\mathbb{X}=\left\{p_{1}, \ldots, p_{s}\right\} \subset[-1,1]^{n} \subset \mathbb{R}^{n}$ be a set of points as above, and let $\sigma$ be a degree compatible term ordering. Consider the following sequence of instructions.

A1 Start with lists $G=\emptyset, \mathcal{O}=(1)$, a matrix $\mathcal{M}=(1, \ldots, 1)^{\mathrm{tr}} \in \operatorname{Mat}_{s, 1}(\mathbb{R}), d=$ 1 , and the list $L=\left(t_{1}, \ldots, t_{\ell}\right)$ of all terms of degree 1 , ordered decreasingly w.r.t. $\sigma$.

A2 Repeat the following steps $\mathbf{A 3}-\mathbf{A} 8$ until $L=\emptyset$. Then return the pair $(\mathcal{O}, G)$ and stop.

A3 Form the matrix $\mathcal{A}=\left(\operatorname{eval}\left(t_{1}\right), \ldots, \operatorname{eval}\left(t_{\ell}\right), \mathcal{M}\right)$ of size $s \times\left(\ell+m^{\prime}\right)$ where $m^{\prime}$ is the number of columns of $\mathcal{M}$. Calculate a matrix $\mathcal{B}$ whose rows are an $O N B$ of the approximate kernel $\operatorname{apker}(\mathcal{A}, \varepsilon)$ of $\mathcal{A}$. Let $k$ be the number of rows of $\mathcal{B}$. Repeat the following steps $\mathbf{A} \mathbf{4}-\mathbf{A} 7$ until $\mathcal{B}=\emptyset$. Then continue with step $\mathbf{A} 8$.

A4 Compute the stabilized reduced row echelon form of $\mathcal{B}$ with respect to the given $\tau$. The result is a matrix $\mathcal{C}=\left(c_{i j}\right) \in \operatorname{Mat}_{k, \ell+m^{\prime}}(\mathbb{R})$ such that $c_{i j}=0$ for $j<\nu(i)$. Here $\nu(i)$ denotes the column index of the pivot element in the $i^{\text {th }}$ row of $\mathcal{C}$.

A5 For all $j \in\{1, \ldots, \ell\}$ such that there exists an $i \in\{1, \ldots, k\}$ with $\nu(i)=j$, append the polynomial

$$
c_{i j} t_{j}+\sum_{j^{\prime}=j+1}^{\ell} c_{i j^{\prime}} t_{j^{\prime}}+\sum_{j^{\prime}=\ell+1}^{\ell+m^{\prime}} c_{i j^{\prime}} u_{j^{\prime}}
$$

to the list $G$, where $u_{j^{\prime}}$ is the $\left(j^{\prime}-\ell\right)^{\text {th }}$ element of $\mathcal{O}$.

A6 For all $j=\ell, \ell-1, \ldots, 1$ such that the $j^{\text {th }}$ column of $\mathcal{C}$ contains no pivot element, append the term $t_{j}$ as a new first element to $\mathcal{O}$ and append the column eval $\left(t_{j}\right)$ as a new first column to $\mathcal{M}$.

A7 Calculate a matrix $\mathcal{B}$ whose rows are an $O N B$ of $\operatorname{apker}(\mathcal{M}, \varepsilon)$.

A8 Increase $d$ by one, let $L=\left(t_{1}, \ldots, t_{\ell}\right)$ be the list of all terms of degree $d$ in $\partial \mathcal{O}$, ordered decreasingly w.r.t. $\sigma$, and continue.

This is an algorithm which computes a pair $(\mathcal{O}, G)$ such that the following properties hold for the bounds $\delta$ and $\eta$ given in [5], Thm. 3.3.

(a) The set $G$ consists of unitary polynomials which vanish $\delta$-approximately at the points of $\mathbb{X}$.

(b) The set $\mathcal{O}=\left\{t_{1}, \ldots, t_{\mu}\right\}$ contains an order ideal of terms such that there is no unitary polynomial in $\langle\mathcal{O}\rangle_{K}$ which vanishes $\varepsilon$-approximately on $\mathbb{X}$.

(c) The set $\widetilde{G}=\left\{\left(1 / \mathrm{LC}_{\sigma}(g)\right) g \mid g \in G\right\}$ is an $\mathcal{O}$-border prebasis.

(d) The set $\widetilde{G}$ is an $\eta$-approximate border basis. 
Our main algorithm combines the techniques of this AVI-algorithm with the subideal version of the BM-algorithm presented above (see Algorithm 4.2). The result is an algorithm which computes an approximate subideal border basis. This notion is defined as follows.

Definition 5.3. Let $\mathcal{O}_{F}=\left\{t_{1} f_{\alpha_{1}}, \ldots, t_{\mu} f_{\alpha_{\mu}}\right\}$ be an $F$-order ideal, let $\partial \mathcal{O}_{F}=$ $\left\{b_{1} f_{\beta_{1}}, \ldots, b_{\nu} f_{\beta_{\nu}}\right\}$ be its border, and let $G=\left(g_{1}, \ldots, g_{\nu}\right)$ be an $\mathcal{O}_{F}$-subideal border prebasis. Recall that this means that $g_{j}$ is of the form $g_{j}=b_{j} f_{\beta_{j}}-\sum_{i=1}^{\mu} c_{i j} t_{i} f_{\alpha_{i}}$ with $c_{i j} \in \mathbb{R}$.

For every pair $(i, j)$ such that $b_{i}, b_{j}$ are neighbors in $\partial \mathcal{O}_{F}$, i.e., such that $\beta_{i}=\beta_{j}$ and $b_{i}, b_{j}$ are neighbors in the usual sense, we compute the normal remainder $S_{i j}^{\prime}=$ $\mathrm{NR}_{\mathcal{O}_{F}, G}\left(S_{i j}\right)$ of the S-polynomial of $g_{i}$ and $g_{j}$ with respect to $G$. We say that $G$ is an $\varepsilon$-approximate $\mathcal{O}_{F}$-subideal border basis if we have $\left\|S_{i j}\right\|<\varepsilon$ for all such pairs $(i, j)$.

Now we are ready to formulate and prove the main result of this section.

Algorithm 5.4 (Subideal Version of the AVI-Algorithm). Let $\mathbb{X}=\left\{p_{1}, \ldots, p_{s}\right\} \subset$ $[-1,1]^{n} \subset \mathbb{R}^{n}$ be a set of points as above, let $\sigma$ be a degree compatible term ordering, and let $F=\left\{f_{1}, \ldots, f_{m}\right\} \subset P \backslash\{0\}$ be a set of \|\|$_{1}$-unitary polynomials which generate an ideal $J=\langle F\rangle$. Consider the following sequence of instructions.

SA1 Let $d=\min \left\{\operatorname{deg}\left(f_{1}\right), \ldots, \operatorname{deg}\left(f_{m}\right)\right\}, \mathcal{O}_{F}=\emptyset, G=\emptyset$, and $\mathcal{M} \in \operatorname{Mat}_{s, 0}(K)$. Let $L=\left(t_{1} f_{\alpha_{1}}, \ldots, t_{\ell} f_{\alpha_{\ell}}\right)$ be the tuple of all $F$-terms of degreed in $F \cup \partial \mathcal{O}_{F}$, with their leading terms ordered decreasingly w.r.t. $\sigma$.

SA2 Repeat the following steps $\mathbf{S A 3 - S A 8 ~ u n t i l ~ w e ~ h a v e ~} L=\emptyset$ and the inequality $d \geq \max \left\{\operatorname{deg}\left(f_{1}\right), \ldots, \operatorname{deg}\left(f_{m}\right)\right\}$. Then return $\left(\mathcal{O}_{F}, G\right)$ and stop.

SA3 Form the matrix $\mathcal{A}=\left(\operatorname{eval}\left(t_{1} f_{\alpha_{1}}\right)|\cdots| \operatorname{eval}\left(t_{\ell} f_{\alpha_{\ell}}\right) \mid \mathcal{M}\right)$ of size $s \times\left(\ell+m^{\prime}\right)$ where $m^{\prime}$ is the number of columns of $\mathcal{M}$. Compute a matrix $\mathcal{B}$ whose rows are an $O N B$ of the approximate kernel of $\mathcal{A}$. Let $k$ be the number of rows of $\mathcal{B}$. Repeat the following steps $\mathbf{S A 4 - S A 7}$ until $\mathcal{B}=\emptyset$. Then continue with step $\mathbf{S A 8 .}$

SA4 Compute the stabilized reduced row echelon form of $\mathcal{B}$ with respect to the given $\tau$. The result is a matrix $\mathcal{C}=\left(c_{i j}\right) \in \operatorname{Mat}_{k, \ell+m^{\prime}}(\mathbb{R})$ such that $c_{i j}=0$ for $j<\nu(i)$. Here $\nu(i)$ denotes the column index of the pivot element in the $i^{\text {th }}$ row of $\mathcal{C}$.

SA5 For all $j \in\{1, \ldots, \ell\}$ such that there exists an $i \in\{1, \ldots, k\}$ with $\nu(i)=j$, append the polynomial

$$
t_{j} f_{\alpha_{j}}+\sum_{j^{\prime}=j+1}^{\ell} c_{i j^{\prime}} t_{j^{\prime}} f_{\alpha_{j^{\prime}}}+\sum_{j^{\prime}=\ell+1}^{\ell+m^{\prime}} c_{i j^{\prime}} u_{j^{\prime}}
$$

to the tuple $G$, where $u_{j^{\prime}}$ is the $\left(j^{\prime}-\ell\right)^{\text {th }}$ element of $\mathcal{O}_{F}$.

SA6 For all $j=\ell, \ell-1, \ldots, 1$ such that the $j^{\text {th }}$ column of $\mathcal{C}$ contains no pivot element, append the $F$-term $t_{j} f_{\alpha_{j}}$ as a new first element to $\mathcal{O}_{F}$, append the column eval $\left(t_{j} f_{\alpha_{j}}\right)$ as a new first column to $\mathcal{M}$.

SA7 Calculate a matrix $\mathcal{B}$ whose rows are an $O N B$ of $\operatorname{apker}(\mathcal{M}, \varepsilon)$.

SA8 Increase $d$ by one. Let $L=\left(t_{1} f_{\alpha_{1}}, \ldots, t_{\ell} f_{\alpha_{\ell}}\right)$ be the tuple of all F-terms of degree $d$ in $F \cup \partial \mathcal{O}_{F}$, with their leading terms ordered decreasingly w.r.t. $\sigma$, and continue.

This is an algorithm which computes a pair $\left(\mathcal{O}_{F}, G\right)$ with the following properties: 
(a) The tuple $G$ consists of unitary polynomials which vanish $\delta$-approximately at the points of $\mathbb{X}$. Here we can use $\delta=\varepsilon \sqrt{\nu}+\tau \nu(\mu+\nu) \sqrt{s}$ with $\mu=\# \mathcal{O}_{F}$ and $\nu=\# G$.

(b) The set $\mathcal{O}_{F}$ contains an $F$-order ideal such that there is no unitary polynomial in $\left\langle\mathcal{O}_{F}\right\rangle_{K}$ which vanishes $\varepsilon$-approximately on $\mathbb{X}$.

(c) The tuple $\widetilde{G}=\left(\left(1 / \mathrm{LC}_{\sigma}(g)\right) g \mid g \in G\right)$ is an $\mathcal{O}_{F}$-subideal border prebasis.

(d) The tuple $\widetilde{G}$ is an $\eta$-approximate subideal border basis for $\eta=2 \delta+2 \nu \delta^{2} / \gamma \varepsilon+$ $2 \nu \delta \sqrt{s} / \varepsilon$. Here $\gamma$ denotes the smallest absolute value of the border F-term coefficient of one the polynomials $g_{i}$.

Proof. Large parts of this proof correspond exactly to the proof of the usual AVIalgorithm (see Thm. 3.2 in [5]). Therefore, we will mainly point out the additional arguments necessary to show the subideal version. The finiteness proof is identical to the finiteness proof in the subideal version of the BM-Algorithm 4.2.

For the proof of (a), we can proceed exactly as in the case of the usual AVIalgorithm. There is only one point where we have to provide a further argument: the norm of the evaluation vector of an $F$-term is $\leq \sqrt{s}$. To see this, we let $t_{i} f_{j}$ be an $F$ term and we write $t_{i} f_{j}=\sum_{k} c_{k} \tilde{t}_{k}$ with $c_{k} \in \mathbb{R}$ and $\tilde{t}_{k} \in \mathbb{T}^{n}$. Since $f_{j}$ is \|\|$_{1}$-unitary and $\mathbb{X} \in[-1,1]^{n}$, we have $\left\|\operatorname{eval}\left(t_{i} f_{j}\right)\right\| \leq \sum_{k}\left|c_{k}\right|\left\|\operatorname{eval}\left(\tilde{t}_{k}\right)\right\| \leq\left\|f_{j}\right\|_{1} \sqrt{s}=\sqrt{s}$.

Next we show (b). The columns of the final matrix $\mathcal{M}$ are precisely the evaluation vectors of the $F$-terms in $\mathcal{O}_{F}$. After the loop in steps SA4-SA7, we have $\operatorname{apker}(\mathcal{M})=\{0\}$. Hence no unitary polynomial in $\left\langle\mathcal{O}_{F}\right\rangle_{K}$ has an evaluation vector which is smaller than $\varepsilon$. It remains to show that $\mathcal{O}_{F}$ is an $F$-order ideal. Suppose that $t_{i} f_{j} \in \mathcal{O}_{F}$ and that $x_{k} t_{i} f_{j}$ is put into $\mathcal{O}_{F}$. We have to prove that every $F$-term $\tilde{t} f_{j}$ such that $x_{\ell} \tilde{t} f_{j}=x_{k} t_{i} f_{j}$ is also contained in $\mathcal{O}_{F}$. In this case we have $t_{i}=x_{\ell} t^{\prime}$ and we want to show $x_{k} t^{\prime} f_{j} \in \mathcal{O}_{F}$. For a contradiction, suppose that $x_{k} t^{\prime} f_{j}$ is the border $F$-term of some $g \in G$. Since the evaluation vector of $x_{\ell} x_{k} t^{\prime} f_{j}=x_{k} t_{i} f_{j}$ is not larger than eval $\left(x_{k} t^{\prime} f_{j}\right)$, also this $F$-term would be detected by the loop of steps SA4-SA7 as the border $F$-term of an element of $G$. This contradicts $x_{k} t_{i} f_{j} \in \mathcal{O}_{F}$.

To prove (c), it suffices to note that the definition of $L$ and step SA5 make sure that the entries of $G$ have the necessary form. Finally, claim (d) follows in exactly the same way as part (d) of [5], Thm. 3.3.

Let us follow the steps of this algorithm in a concrete case which is a slightly perturbed version of Example 4.3.

Example 5.5. In the ring $P=\mathbb{R}[x, y, z]$ we consider the ideal $J=\left\langle f_{1}, f_{2}\right\rangle$ generated by the \|\|$_{1}$-unitary polynomials $f_{1}=0.5 y-0.5 z$ and $f_{2}=0.5 x^{2}-0.5$. Let $\sigma=$ DegRevLex, let $\varepsilon=0.03$, and let $\tau=0.001$. We want to compute an approximate subideal border basis vanishing approximately at the points of $\mathbb{X}=\{(1,1,1),(0,1,1),(1,1,0),(1,0,0.98),(0.98,0,1)\}$.

Notice that the first point of $\mathbb{X}$ is contained in $\mathcal{Z}\left(f_{1}, f_{2}\right)$ and that the last two points of $\mathbb{X}$ differ by $\leq \varepsilon$ from one point $(1,0,1)$. Hence the approximate subideal border basis should correspond to three points outside $\mathcal{Z}(J)$, and therefore we should expect to get an $F$-order ideal consisting of three $F$-terms. We follow the steps of the subideal version of the AVI-Algorithm 5.4.

SA1 Let $d=1$ and $L=(0.5 y-0.5 z)$.

SA3 We compute $\mathcal{A}=(0,0,0.5,-0.49,-0.51)^{\mathrm{tr}}$ and $\mathcal{B}=(0)$. (Thus $\mathcal{C}=\mathcal{B}$.)

SA6 Let $\mathcal{O}=\left\{f_{1}\right\}$ and $\mathcal{M}=(0,0,0.5,-0.49,-0.5)^{\operatorname{tr}}$. 
SA8 Let $d=2$ and $L=\left(f_{2}, x f_{1}, y f_{1}, z f_{1}\right)$.

SA3 We compute $\mathcal{A}=\left(\begin{array}{ccccc}0 & 0 & 0 & 0 & 0 \\ -0.5 & 0 & 0 & 0 & 0 \\ 0 & 0.5 & 0.5 & 0 & 0.5 \\ 0 & -0.49 & 0 & -0.4802 & -0.49 \\ -0.0198 & -0.49 & 0 & -0.5 & -0.5\end{array}\right)$ and

$$
\mathcal{B}=\operatorname{apker}(\mathcal{A}, \varepsilon)=\left(\begin{array}{ccccc}
0.0004 & 0.6755 & -0.5089 & -0.5068 & -0.1667 \\
0 & -0.3812 & -0.3735 & -0.3812 & 0.7548
\end{array}\right) .
$$

SA4 The stabilized reduced row echelon form of $\mathcal{B}$ is

$$
\mathcal{C}=\left(\begin{array}{ccccc}
0 & 0.7070 & 0 & 0.0074 & -0.7071 \\
0 & 0 & 0.5754 & 0.5811 & -0.5754
\end{array}\right)
$$

SA5 We get $G=\left(g_{1}, g_{2}\right)$ with $g_{1}=0.707 x f_{1}+0.0074 z f_{1}-0.7071 f_{1}$ and $g_{2}=$ $0.5754 y f_{1}+0.5811 z f_{1}-0.5754 f_{1}$.

SA6 We find $\mathcal{O}=\left\{f_{2}, z f_{1}, f_{1}\right\}$ and $\mathcal{M}=\left(\begin{array}{ccccc}0 & -0.5 & 0 & 0 & 0 \\ 0 & 0 & 0 & -0.4802 & -0.5 \\ 0 & 0 & 0.5 & -0.49 & -0.5\end{array}\right)^{\operatorname{tr}}$.

SA8 Now let $d=3$ and $L=\left(x f_{2}, y f_{2}, z f_{2}, x z f_{1}, y z f_{1}, z^{2} f_{1}\right)$.

SA3 $\mathcal{A}=\left(\begin{array}{ccccccccc}0 & 0 & 0 & 0 & 0 & 0 & 0 & 0 & 0 \\ 0 & -0.5 & -0.5 & 0 & 0 & 0 & -0.5 & 0 & 0 \\ 0 & 0 & 0 & 0 & 0 & 0 & 0 & 0 & 0.5 \\ 0 & 0 & 0 & -0.48 & 0 & -0.47 & 0 & -0.48 & -0.49 \\ -0.02 & 0 & -0.02 & -0.49 & 0 & -0.5 & -0.02 & -0.5 & -0.5\end{array}\right)$

and $\mathcal{B}, \mathcal{C}$ are matrices of rank 6 which yield six further approximate subideal border basis elements.

SA5 We obtain $G=\left(g_{1}, \ldots, g_{8}\right)$ with $g_{3}=x f_{2}-0.02 z f_{1}, g_{4}=0.71 y f_{2}-$ $0.71 f_{2}+0.01 z f_{1}, g_{5}=0.71 z f_{2}-0.71 f_{2}, g_{6}=0.71 x z f_{1}-0.7 z f_{1}, g_{7}=y z f_{1}$, and $g_{8}=0.71 z^{2} f_{1}-0.7 z f_{1}$.

SA6 Since there is no new non-pivot row index, $\mathcal{O}_{F}$ and $\mathcal{M}$ are not changed.

SA8 In degree $d=4$ we find $L=\emptyset$ and the algorithm stops.

Hence the result is the $F$-order ideal $\mathcal{O}=\left\{x^{2}-1, z(y-z), y-z\right\}$ and the approximate $\mathcal{O}_{F}$-subideal border basis $G=\left(g_{1}, \ldots, g_{8}\right)$. This confirms that there are three approximate zeros of $G$ outside the two lines $\mathcal{Z}\left(f_{1}, f_{2}\right)$.

\section{EFFICIENCY AND TIMINGS}

Let us briefly discuss the efficiency of the subideal versions of the BM-algorithm and the AVI-algorithm. Let $K$ be a field, let $P=K\left[x_{1}, \ldots, x_{n}\right]$, let $I \subset P$ be a 0 -dimensional ideal, and let $J \subset P$ be an arbitrary ideal generated by $\left\{f_{1}, \ldots, f_{m}\right\}$. Since no theory of border bases for ideals $(J+I) / I$ in residue class rings of the form $P / I$ is available, the standard approach to compute a subideal border basis seems to be to perform the following steps:

(1) Compute an order ideal $\mathcal{O}$ and an $\mathcal{O}$-border basis of the ideal $I$.

(2) Compute the kernel $U$ of the map $P^{m} \longrightarrow P / I$ given by $e_{i} \mapsto \bar{f}_{i}$.

(3) Determine order ideals $\mathcal{O}_{i} \subseteq \mathcal{O}$ for $i=1, \ldots, m$ such that $\mathcal{O}_{1} e_{1} \cup \cdots \cup \mathcal{O}_{m} e_{m}$ is a $K$-basis of $P^{m} / U$.

For the third step, it appears to be necessary to calculate a Gröbner basis of $U$. Except for the simplest case below, this operation exceeded the capacity of the 
available computer. In these cases we give (in parentheses) the timings for the first two steps only.

In the following table we compare the running times of the implementations in ApCoCoA of this standard approach to the subideal version of the BM-Algorithm 4.2, as well as to the subideal version of the AVI-Algorithm 5.4. In the approximate setting no alternative approach is known to us.

\begin{tabular}{|l|l|l|l|}
\hline point set & std. approach & subideal BM & subideal AVI \\
\hline 10 random points in $\mathbb{R}^{3}$ & 0.7 & 0.09 & 0.015 \\
30 random points in $\mathbb{R}^{3}$ & $(22)$ & 15.6 & 0.14 \\
30 random points in $\mathbb{R}^{5}$ & $(99)$ & 18.7 & 0.6 \\
2445 points in $\mathbb{R}^{9}$ & - & - & 1.6 \\
7400 points in $\mathbb{R}^{8}$ & - & - & 6.4 \\
\hline
\end{tabular}

These timings are measured in seconds. They were obtained using ApCoCoA 1.3 on a small laptop running at $2 \mathrm{Ghz}$ and having $2 \mathrm{~GB}$ of memory. Dashes indicate that the computation was not finished after two hours and had to be terminated unsuccessfully. All points have coordinates in $[-1,1]$. The coordinates of random points are rational numbers with 3 -digit numerators and denominators. The last two examples are based on real world measured data from the oil industry. All timings depend on the complexity of the ideal $J$. In view of the application explained below, the ideal $J$ we used was generated by the first two indeterminates. For the subideal AVI computation we chose the threshold number $\varepsilon=0.1$.

The table shows that the subideal version of the BM-algorithm is much more efficient than the standard approach, even if we skip step (3) of the standard approach. Moreover, the subideal AVI-algorithm is even faster since it can rely on floating point arithmetic and numerical linear algebra methods. Thus it enables us to handle industrial size data sets.

\section{AN INDUSTRIAL APPLICATION}

In this section we apply the subideal version of the AVI-algorithm to an actual industrial problem which has been studied in the Algebraic Oil Research Project (see 2]). Viewed from a more general perspective, this application shows how one can carry out the suggestion made in the introduction, namely to use the subideal version of the AVI-algorithm to introduce knowledge about the nature of a physical system into the modeling process.

Suppose that a multi-zone well consists of two zones $A$ and $B$. During so-called commingled production, the two zones are interacting and influence each other. We have at our disposal time series of measured data such as pressures, temperatures, total production and valve positions. Moreover, during so-called test phases we can obtain time series of these data when only one of the two zones is producing. The following figure gives a schematic representation of the physical system and the measured variables.

The measured total production does not equal the sum of the individual productions calculated from the test data. The production allocation problem is to determine the contributions of the two zones to the total production when they are producing together. Here the contributions $c_{A}, c_{B}$ of the zones are defined to be the part of the total production $p_{A B}$ passing through the corresponding down-hole valves. Therefore, we have $p_{A B}=c_{A}+c_{B}$, but there is no way of measuring $c_{A}$ 


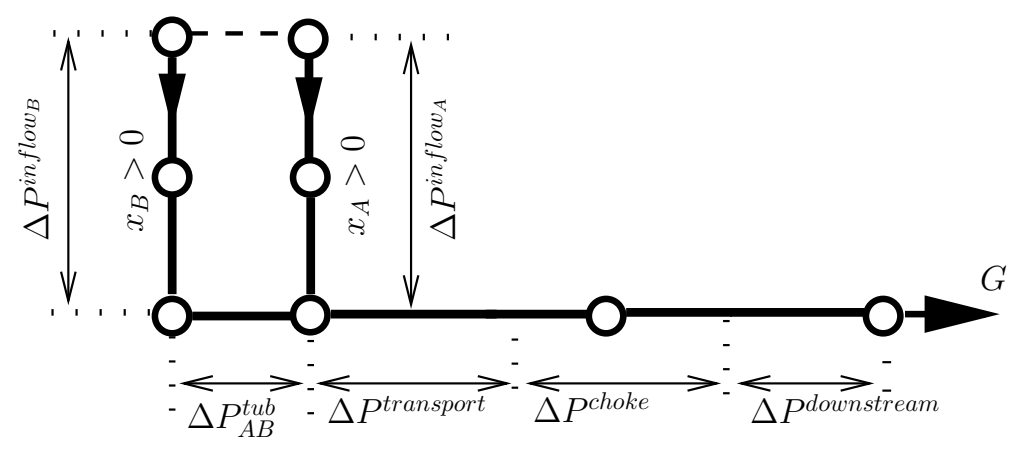

FiguRE 1. Schematic representation of a two-zone well

and $c_{B}$ directly. In this sense the production allocation problem is to determine the contributions $c_{A}, c_{B}$ from the measured data.

Let the indeterminate $x_{A}$ represent the valve position of zone $A$ and $x_{B}$ the valve position of zone $B$. Here $x_{i}=0$ means that the valve is closed and $x_{i}=1$ represents a fully opened valve position. Clearly, if valve $A$ is closed, i.e., for points in the zero set $\mathcal{Z}\left(\left\langle x_{A}\right\rangle\right)$, there is no contribution from zone $A$, and likewise for $B$. By Hilbert's Nullstellensatz, this means that the polynomial $p_{A}$ modeling the production of zone $A$ should be computed by using the subideal version of the AVI-algorithm with $J=\left\langle x_{A}\right\rangle$. Similarly, we want to force $p_{B} \in\left\langle x_{B}\right\rangle$.

Now we model the total production $p_{A B}$ in the following way. We write $p_{A B}=$ $p_{A}+p_{B}+q_{A B}$ where $q_{A B}$ is a polynomial which measures the interaction of the two zones. To compute $q_{A B}$, we write it in the form

$$
q_{A B}=f_{A} \cdot\left(x_{B} \cdot p_{A}\right)+f_{B} \cdot\left(x_{A} \cdot p_{B}\right) .
$$

Notice that such a decomposition can be computed via the subideal version of the AVI-algorithm by applying it to the ideal $J=\left\langle x_{B} p_{A}, x_{A} p_{B}\right\rangle$. The result will be a representation $p_{A B}=p_{A}+p_{B}+f_{A} x_{B} p_{A}+f_{B} x_{A} p_{B}$. Here we observe that $x_{A}=0$ implies $p_{A B}=p_{B}$ because $p_{A} \in\left\langle x_{A}\right\rangle$. Analogously, we see that $x_{B}=0$ implies $p_{A B}=p_{A}$, in accordance with the physical situation.

The end result of these computations is that the contributions of the two zones during comingled production can be computed from the equalities $c_{A}=(1+$ $\left.f_{A} x_{B}\right) p_{A}$ and $c_{B}=\left(1+f_{B} x_{A}\right) p_{B}$. At the same time we gain a detailed insight into the nature of the interactions by examining the structure of the polynomials $f_{A}, f_{B}$.

\section{ACKNOWLEDGEMENTS}

The idea to construct a subideal version of the AVI-algorithm originated in discussions of the authors with Daniel Heldt who also implemented a rough first prototype. The algorithms of this paper have been implemented by Jan Limbeck in the ApCoCoA library (see [3]) and are freely available. The authors thank both of them for the opportunity to use these implementations in the preparation of this paper and in the Algebraic Oil Research Project (see [2]). The support of Shell Research (Rijswijk, NLD) to the Algebraic Oil Research Project is greatly appreciated. Special thanks go to Lorenzo Robbiano for useful discussions and to the Dipartimento di Matematica of Università di Genova (Italy) for the hospitality 
the authors enjoyed during part of the writing of this paper. We are indebted to Dmitry Pavlov for point out an error in a previous version of this paper.

\section{REFERENCES}

[1] J. Abbott, C. Fassino, and M. Torrente, Stable border bases for ideals of points, J. Symb. Comput. 43 (2008), 883-894. MR2472538(2010a:13043)

[2] The Algebraic Oil Research Project, see http://www.fim.uni-passau.de/algebraic-oil

[3] ApCoCoA: Applied Computations in Commutative Algebra, see http://www.apcocoa.org

[4] B. Buchberger and H. M. Möller, The construction of multivariate polynomials with preassigned zeros, in: J. Calmet (ed.), Proceedings of EUROCAM'82, Lect. Notes in Comp. Sci. 144, Springer, Heidelberg, 1982, 24-31. MR680050 (84b:12003)

[5] D. Heldt, M. Kreuzer, S. Pokutta and H. Poulisse, Approximate computation of zerodimensional polynomial ideals, J. Symb. Comput. 44 (2009), 1566-1591. MR2561289

[6] A. Kehrein and M. Kreuzer, Characterizations of border bases, J. Pure Appl. Alg. 196 (2005), 251-270. MR2116166 (2006e:13037)

[7] A. Kehrein, M. Kreuzer and L. Robbiano, An algebraist's view on border bases, in: A. Dickenstein and I. Emiris (eds.), Solving polynomial equations, Alg. and Comput. in Math. 14, Springer-Verlag, Heidelberg, 2005, pp. 169-202. MR2161988

[8] M. Kreuzer, H. Poulisse, and L. Robbiano, From oil fields to Hilbert schemes, in: J. Abbott, L. Robbiano (eds.), Approximate Commutative Algebra, Springer-Verlag, Vienna, 2009, pp. $1-54$.

[9] M. Kreuzer and L. Robbiano, Computational Commutative Algebra 1, Springer-Verlag, Heidelberg, 2000. MR1790326 (2001j:13027)

[10] M. Kreuzer and L. Robbiano, Computational Commutative Algebra 2, Springer-Verlag, Heidelberg, 2005. MR2159476 (2006h:13036)

[11] M. Kreuzer and L. Robbiano, Deformations of border bases, Collect. Math. 59 (2008), 275297. MR2452308 (2010a:13046)

[12] K. Shirayanagi, M. Sweedler, Remarks on automatic algorithm stabilization, J. Symb. Comput. 26 (1998), 761-765. MR1662034 (99h:65086)

[13] H. Stetter, Numerical Polynomial Algebra, SIAM, Philadelphia, 2004. MR2048781 (2006a:65004)

Fakultät für Informatik und Mathematik, Universität Passau, D-94030 Passau, GerMANY

E-mail address: martin.kreuzer@uni-passau.de

Harkenkamp 1A, D-30851 Langenhagen, Germany

E-mail address: henk.poulisse@gmail.com 